Check for updates

Cite this: J. Mater. Chem. A, 2021, 9 , 27170

Received 29th September 2021 Accepted 5th November 2021

DOI: $10.1039 / \mathrm{d} 1 \mathrm{ta0} 8424 \mathrm{k}$

rsc.li/materials-a

\section{Naphthalene diimide-based electron transport materials for perovskite solar cells}

\author{
Mohammed A. Jameel, (D) ab Terry Chien-Jen Yang, (D) +t. Gregory J. Wilson, (D)*b \\ Richard A. Evans, (D) ${ }^{c}$ Akhil Gupta (D)*acd and Steven J. Langford (D) *a
}

The development of perovskite solar cells (PSCs) as an efficient and cost-effective alternative to traditional approaches to solar energy transduction has received much recent attention, and there has been considerable progress made with reported power conversion efficiencies now surpassing $25 \%$. This development is encouraging and is a result of intensive research on device design, factors affecting long-term stability of PSCs, and systematic material development in which electron transport layer (ETL) materials play a crucial role to afford high-performance PSC devices. ETL materials, including (6-(1,10phenanthrolin-3-yl)naphthalen-2-yl)diphenylphosphine oxide (Phen-NaDPO), and n-type materials based on the naphthalene diimide (NDI) structure, appear to be amongst the most promising materials to date. This article provides an up-to-date review on organic n-type ETL materials, both polymeric and small molecules, based on NDI format, detailing reports of structures with key relevant parameters, such as the efficiency and stability of PSCs. The review is written from a perspective of organic chemistry and we believe this will provide fundamental advice on the future design of new ETL materials based on NDIs that will afford more efficient and stable PSCs.
${ }^{a}$ Department of Chemistry and Biotechnology, School of Science, Computing and Engineering Technology, Swinburne University of Technology, Hawthorn, Victoria 3122, Australia. E-mail: sjlangford@swin.edu.au; Tel: +61 392143732

${ }^{b}$ CSIRO Energy, 10 Murray Dwyer Circuit, Mayfield West, NSW 2304, Australia. E-mail: gregory.wilson@csiro.au

${ }^{\circ}$ CSIRO Manufacturing, Bayview Avenue, Clayton South, Victoria 3169, Australia
${ }^{d}$ Institute for Frontier Materials, Deakin University, Waurn Ponds, Victoria 3216, Australia.E-mail: a.gupta@deakin.edu.au

$\dagger$ Present address: Department of Chemical Engineering and Biotechnology, University of Cambridge, Cambridge, UK.

$\ddagger$ Present address: Cavendish Laboratory, Department of Physics, University of Cambridge, Cambridge, UK.

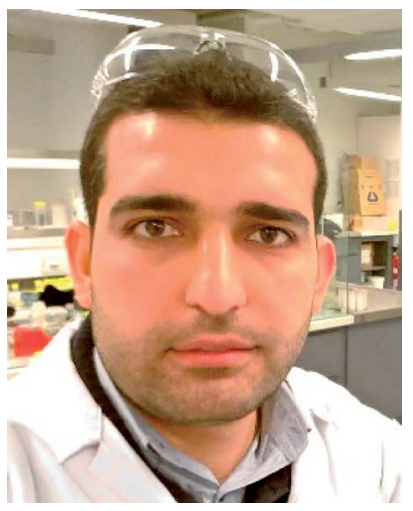

Mohammed A. Jameel is a PhD researcher (2019-present) in Chemistry, at Swinburne University, Hawthorn, Australia. His doctoral project, supported by Swinburne University and CSIRO Energy in Newcastle, Australia, focuses on the design and characterisation of NDIbased active materials for organic and perovskite solar cells. He received his master's degree in science from University of Duhok, Kurdistan, Iraq, in collaboration with the University of Melbourne, Parkville, Australia (2017). He has experience of over a decade of senior demonstrative roles teaching chemistry and laboratory skills.

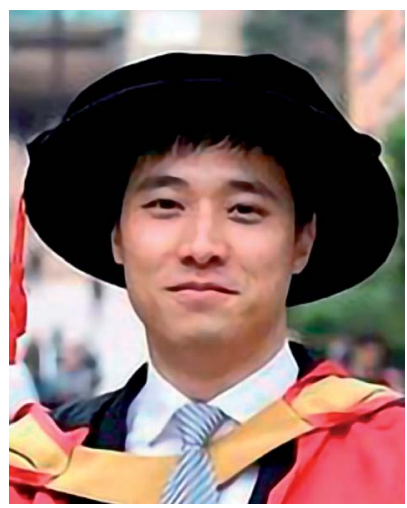

Dr Terry Chien-Jen Yang received his $P h D$ in Photovoltaic Engineering (2016) and BEng in Photovoltaic and Solar Energy Engineering with 1st Class Hons. (2011) from UNSW in Sydney, Australia. He recently joined StranksLab at the University of Cambridge as a new Marie Skłodowska-Curie postdoc fellow working on lightweight and flexible allperovskite triple-junction solar cells. Previously, he worked on perovskite-silicon tandem solar cells as a postdoctoral research fellow at CSIRO Energy in Newcastle, Australia (2019-2021) and as a MSCA fellow at PV-Lab, EPFL in Switzerland (2017-2019). 


\section{Introduction}

It is predicted that 2040 world energy demand will be around 18 million tonnes of oil equivalent (Mtoe), a 26\% increase when compared to 2017..$^{1}$ Within this prediction, the demand for electricity increases by $2.1 \%$ per year - which is double the rate of demand for all forms of energy. ${ }^{2}$ To cope with that demand, and to reduce our reliance on fossil fuels, sustainable renewable energy sources have been identified that are not only abundant, but can be utilized for centuries to come. Natural or renewable energy sources such as solar, wind, tidal, and biomass energy are appraised to be the primary sources to solve the energy crisis in the short to medium term. Solar energy, in particular, is the most powerful, continuous, and feasible source, and its availability by far exceeds any potential future energy demand. ${ }^{3}$ The strong rationale behind this logic is the abundant incoming solar power [89 petawatt, $\left(1\right.$ petawatt $=10^{15}$ watts $)$, that falls upon the planet's surface - enough energy every hour to supply societies' energy demands for a year (Fig. 1). ${ }^{3-5}$ It is not possible

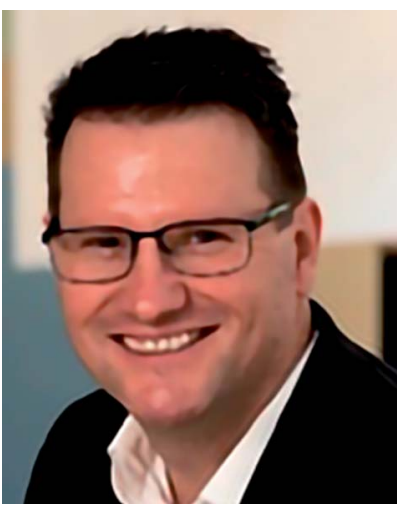

Prof. Gregory Wilson is Senior Principal Research Scientist and Research Group Leader for Solar Technologies in CSIRO Energy and Conjoint Professor at University of Newcastle. Prof. Wilson obtained his Bachelor of Science (2000), First Class Honours (2001), and Ph.D. (2006) degrees from Queensland University of Technology. He joined CSIRO in 2005. Gregory's research focuses on thin-film materials, solution processed thin-film photovoltaics, and mesoscopic solar cells.

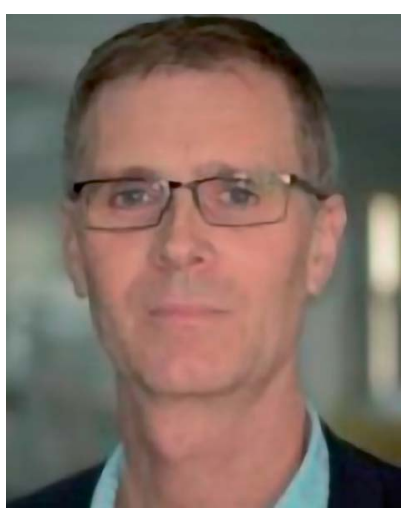

Dr Richard Evans received his PhD in Organic Chemistry in 1992. He subsequently joined CSIRO in Melbourne and is currently a Chief Research Scientist and Senior Project Leader. His research interests are in the development of highly functional materials using living radical polymerization methods with stimuli responsiveness being a recurring theme. He has published and patented in the research areas of click chemistry, non-linear dyes, dyes and hole transport materials for organic solar cells, photochromism, new paint resins, low shrinkage ring-opening polymerization, and functional ATRP and RAFT agents. to harness all, or even most of this energy, yet capturing less than $0.02 \%$ would be enough to meet the current global energy needs. Given solutions to energy storage need to be able to supply base load power, environmental and commercial advantages with the use of solar technology include a noise and pollution free environment, non-toxic solar panels, no greenhouse gas emission, very little and low-cost year-on-year maintenance, an ease of integration into our built environment and grid independence, should also offer the technology to be used in remote areas and as a mobile energy source. Moreover, the practical potential of solar electricity makes it attractive for large-scale utilization. The last decade has witnessed a continuous increase in the intensive research efforts that have been carried out to develop various solar cell technologies with the aims of increasing the power conversion efficiency, reducing the cost, and improving their lifetime in the field.

The photovoltaic (PV) effect describes the generation of an electric current and voltage in a material incident with radiation (predominately light) and is a physical and chemical

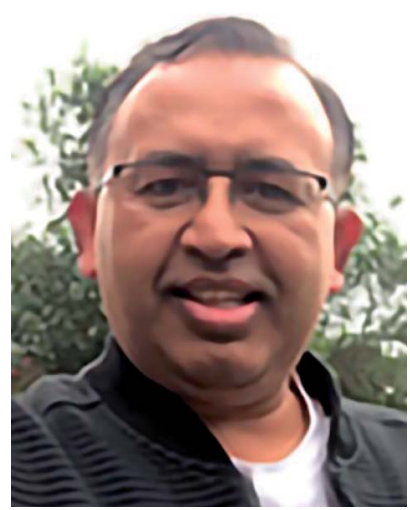

Dr Akhil Gupta obtained his PhD from Monash University (Australia) and has held research positions at CSIRO Australia, Monash and Deakin Universities, the University of $\mathrm{Mel}$ bourne, and Swinburne University of Technology. He is currently a research fellow at the Institute for Frontier Materials, Deakin University, Australia. Concurrently, he is a visiting research fellow at Swinburne University of Technology and CSIRO, Australia. His research interests are in the design and development of $\pi$-functional materials for organic and perovskite solar cells, chemical sensing, self-assembly, and supramolecular chemistry.

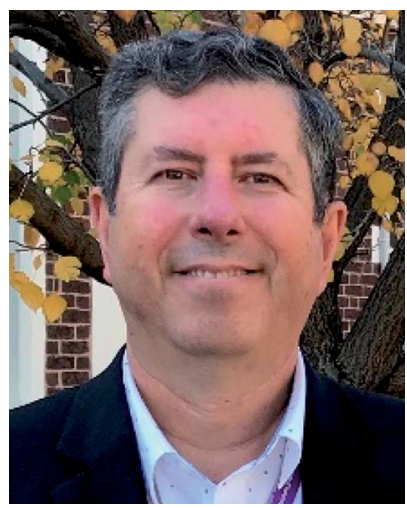

Prof Steven Langford completed his PhD in 1994 from the University of Sydney and was the sole recipient of the Ramsay Memorial Trust Fellowship working with Prof Fraser Stoddart (2016 Chemistry Nobel Prize winner). After an $A R C$ postdoc Fellowship at UNSW, he moved to Monash to begin his independent career in 1998, becoming Professor in 2006 and Head of School in 2010. After a distinguished career at Monash, he joined Swinburne in October 2017 as Dean, Research and Development. His interests involve the application of organic chemistry to new materials' research, utilising the principles of supramolecular chemistry. 


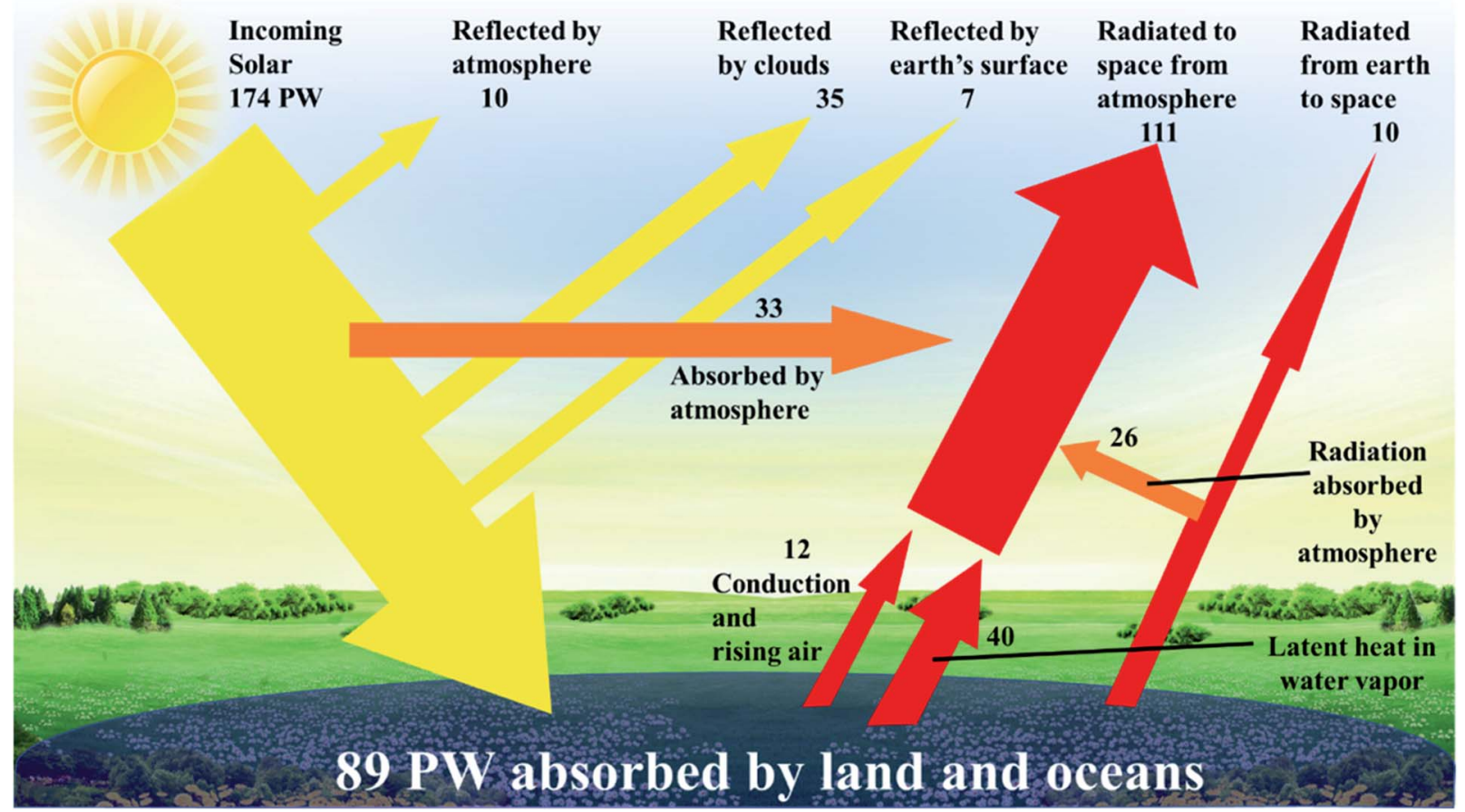

Fig. 1 Incoming solar power distribution is reflected and absorbed in many ways. ${ }^{5}$

phenomenon. The PV effect was first observed by French physicist, A.E. Becquerel, in 1839 while studying liquid electrolytes. ${ }^{6}$ From the 1950s, this effect has been studied with a wide range of materials and different device architectures ever since the discovery of Chapin that a silicon-based, single $p-n$ junction device with a solar power conversion efficiency (PCE) of approximately $6 \%$ was achieved in $1954 .^{7} \mathrm{PV}$ technology is currently dominated by wafer-sized single junction solar cells based on crystalline silicon that are assembled into large area modules. These first-generation solar cells have dominated the PV market due to their relative low-cost and large-scale manufacturing capability. ${ }^{8,9}$

Today, the most successful crystalline silicon solar cell has been the passivated emitter and rear cell (PERC) ${ }^{10}$ pioneered by M.A. Green and his team since the 1980 s. $^{11}$ However, due to the limitations of single-junction crystalline silicon solar cells, including finite photon conversion and rigid nature, several alternatives have been proposed such as thin film devices based on cadmium telluride, copper indium gallium selenide, and amorphous silicon. Thin-film technologies, which require less materials to form devices, are known as second generation PV solar cells. Even though these second-generation devices can be less expensive with respect to materials, production costs can be higher, and they are still lower in overall light to electrical PCE than their first-generation counterparts. Simultaneously, organic semiconducting materials have advanced very rapidly to provide a range of organic-based devices, such as organic PV cells, since Tang reported a single heterojunction organic PV cell with PCE of $0.95 \% .^{12}$ This work represented a major milestone for the development of organic-based semiconductor PV devices. From the late ' 80 s to early ' 90 s, high-purity conjugated polymers paved the way for the fabrication of organic PV devices and these cells - addressing further cost reductions through lower materials costs based on organic materials and processing techniques - are referred to as third-generation PV technologies. ${ }^{13-16}$ In addition to organic heterojunction PV devices, third generation technologies include a variety of other devices, including dye sensitized solar cells (DSSCs), ${ }^{17}$ perovskite solar cells (PSCs) ${ }^{18}$ and advanced technologies such as tandem and multijunction solar cells which allow the solar cell efficiency to surpass those of its single-junction counterparts. ${ }^{19-21}$ Tandem and multijunction solar cells may consist of a single device technology or mixture of technologies from different generations, for example GaInP/GaInAs/Ge, a-Si/nc-Si/nc-Si (thin film), perovskite/silicon, perovskite/CIGS, perovskite/perovskite and more. ${ }^{22}$ These third-generation devices are a promising costeffective alternative to the conventional first-generation silicon-based solar cells as their main advantages include: ease of processing (solution processability), mechanical flexibility and versatility of chemical structures and properties from advances in organic chemistry. ${ }^{23-26}$ However, their commercial viability and production is a considerable challenge for research and industry as they exhibit moderate efficiencies, lower stability and, in some cases, higher cost compared to conventional silicon-based solar cells. There are extensive efforts to solve these issues with the development of organic PV devices, in fact, the widespread commercialisation of PSCs is anticipated in the near future.

Though PV devices can be classified by their fabrication strategies and chronological development over time, a more appealing approach would be to classify PV technology by the nature of their active, or light absorbing material as shown in 


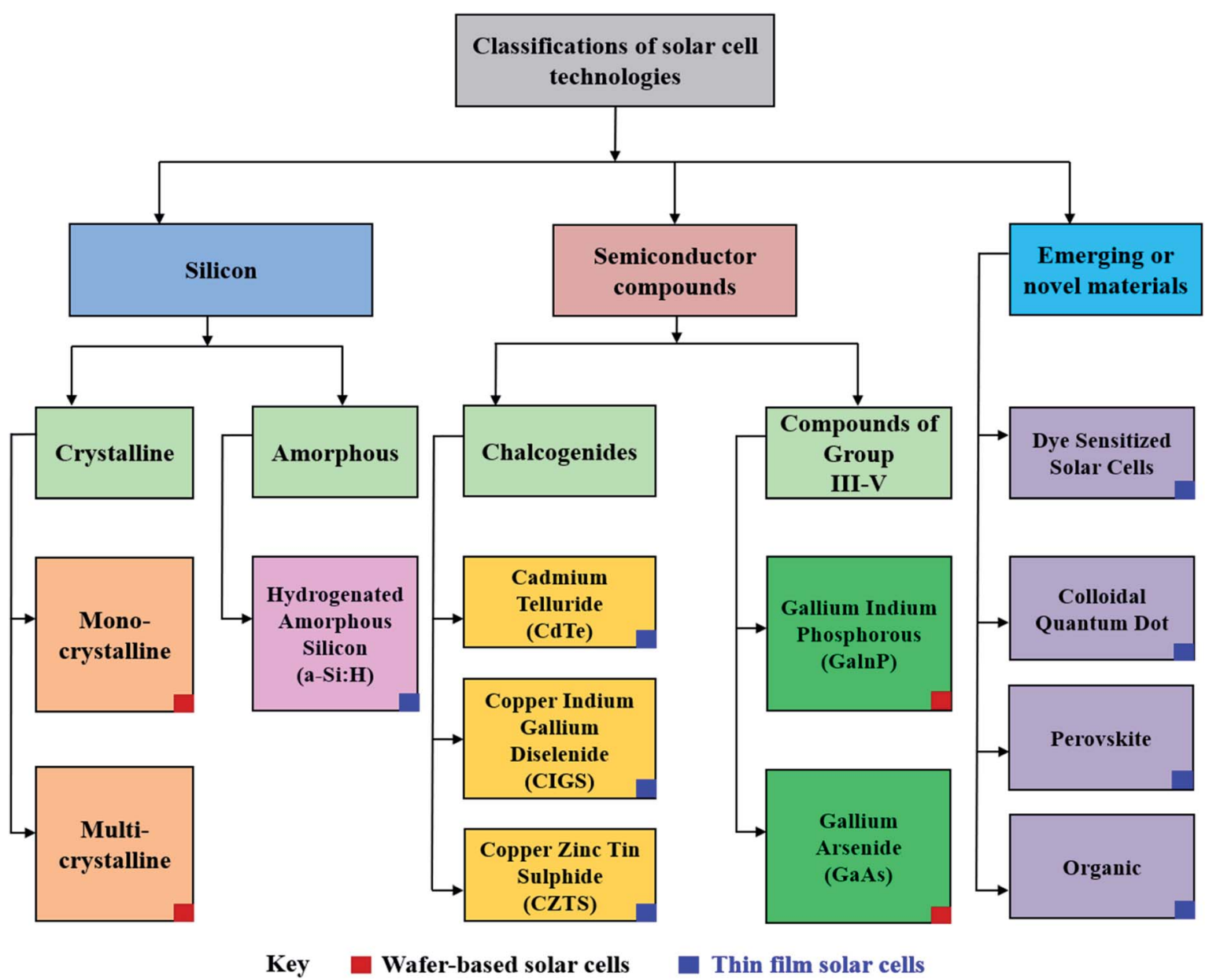

Fig. 2 Alternative classification of solar cells based on the primary light-absorbing material within the device. ${ }^{27}$

Fig. 2. 27,28 Here, active materials form three major classes: silicon-based, inorganic semiconductors, and emerging or novel materials which contain amongst other materials, perovskite.

The simple architecture of a typical PSC device contains three major components. The first comprises an absorbing layer which is an organic-inorganic hybrid halide-based perovskite material of general formula $\mathrm{ABX}_{3}$, where $\mathrm{A}$ and $\mathrm{B}$ are cations of various sizes (typically $\mathrm{A}=\mathrm{CH}_{3} \mathrm{NH}_{3}{ }^{+}, \mathrm{CH}\left(\mathrm{NH}_{2}\right)_{2}{ }^{+}$or $\mathrm{Cs}^{+}$and $\mathrm{B}=$ $\mathrm{Pb}^{2+}$ or $\mathrm{Sn}^{2+}$ ) and $\mathrm{X}$ is a monovalent anion, typically the halide anions, $\mathrm{I}^{-}, \mathrm{Br}^{-}, \mathrm{Cl}^{-}$, and a combination thereof.

The general macromolecular structure is shown in Fig. 3. The second component of a PSC device is the charge-carrying transport layers, which include electron and hole transporting layers that are responsible for extracting and transporting electrons and holes, respectively, from the perovskite layer to the corresponding electrodes. The third component is the electrodes, which comprises of an anode and a cathode. A variety of electrodes, including indium tin oxide (ITO), fluorine tin oxide (FTO), gold $(\mathrm{Au})$, silver $(\mathrm{Ag})$, copper $(\mathrm{Cu})$ and aluminium (Al), have been frequently reported in the literature. ${ }^{29}$ Using these key three components, the most commonly assembled structures for PSC device architectures can be classed as: (1) mesoporous n-i-p (note: the letters n, i, and $p$ stand for n-type layer, perovskite/light-absorbing layer (intrinsic), and p-type layer in a perovskite device geometry), (2) planar $n-i-p$, and (3) inverted p-i-n, as depicted in Fig. 4.

The earliest reports for PSCs reported the use of methylammonium lead iodide and methylammonium lead bromide as
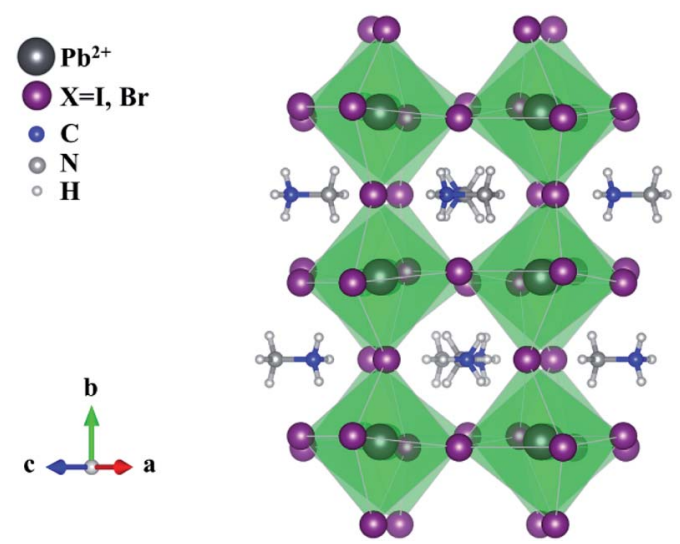

Fig. 3 Structure of a typical perovskite $\mathrm{ABX}_{3}$ crystal structure where $\mathrm{A}$ and $\mathrm{B}$ are cations of different sizes, e.g. $\mathrm{CH}_{3} \mathrm{NH}_{3}{ }^{+}$and $\mathrm{Pb}^{2+}$, and $\mathrm{X}$ is a monovalent anion, e.g. $\mathrm{I}^{-}$or $\mathrm{Cl}^{-}$or $\mathrm{Br}^{-}$. 

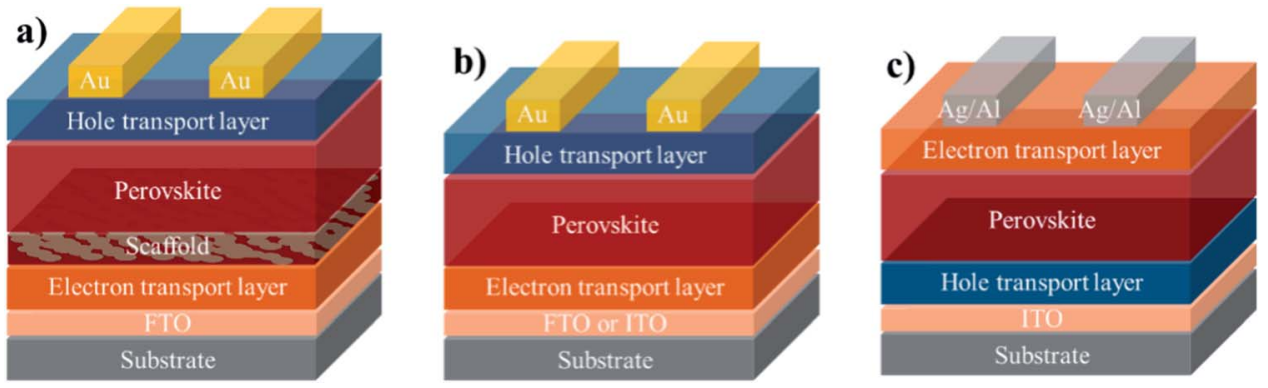

Fig. 4 Examples of commonly used PSC device architectures, (a) mesoporous $n-i-p$, (b) planar $n-i-p$, and (c) inverted p-i-n [note: the letters $n$, $\mathrm{i}$, and $\mathrm{p}$ stand for $\mathrm{n}$-type layer, perovskite/light-absorbing layer (intrinsic), and $\mathrm{p}$-type layer in a typical perovskite device geometry].

the light absorbing materials. ${ }^{30}$ The reported PCE of these as DSSCs was low at $3.8 \%$, yet the use of such organic-inorganic halide materials instigated a new research theme which was explored in terms of new material design and creative device fabrication strategies. In 2011, Park and co-workers reported an increased PCE of $6.5 \%$ for $\mathrm{MAPbI}_{3}$-based devices where they modified both the titania surface and the processing method of depositing the perovskite layer. ${ }^{31}$ In 2012, this idea was advanced further through the seminal reports on solid-state PSCs published by the Snaith and Grätzel groups, which used mixed-halide compositions of the photoactive materials $\left(\mathrm{MAPbI}_{3}\right.$ and $\mathrm{MAPbI}_{3-x} \mathrm{Cl}_{x}$ ) deposited onto a mesoporous $\mathrm{TiO}_{2}$ layer. ${ }^{32}$ In that same year, the Snaith group also reported on a hybrid PSC based on a meso-superstructured organometal halide perovskite. The highly crystalline perovskite absorber, with intense absorptivity of $350 \mathrm{~nm}$ to $\sim 950 \mathrm{~nm}$, was fabricated in solution-processed PSC devices and afforded a PCE of $10.9 \%{ }^{33}$ Since then, research on PSCs has intensified with the most recent reports of PCE of $>25 \%$ challenging the status of silicon as the dominant semiconductor material for low-cost photovoltaics. $^{34,35}$ Importantly, stability of the highest efficiency devices $(>24 \%$ PCE) in humid conditions without encapsulation has improved significantly over time. ${ }^{36}$ It is evident that given the efficiency/fabrication cost ratio of PSCs, this technology is considered more viable for global future energy needs when compared with other third-generation technologies such as OPVs and DSSCs.

\section{Organic electron transport layer materials}

The ETL (sometimes called the electron extraction layer or electron collection layer) is a layer in a given PSC device architecture where the electrons are injected from the absorber layer, transported through this layer, i.e. through the thin film of an ETL material, and finally collected by the electrode. For this to occur efficiently, the energy levels, i.e. the highest occupied molecular orbital (HOMO) (or valence band maximum) and the lowest unoccupied molecular orbital (LUMO) (conduction band minimum), of an ETL material and the perovskite layer should complement each other. Having said that, the ETL material should also have the LUMO and the HOMO levels higher than the perovskite active layer. The ETL material should, ideally, have high transmittance in the UV-Vis region, depending upon the type of device architecture, so that photons can pass through and be absorbed by the perovskite layer. It is evident from the current literature that inorganic metal oxides, such as $\mathrm{TiO}_{2}, \mathrm{SnO}_{2}, \mathrm{ZnO}$ (Fig. 5), are usually preferred as ETL materials, with each material having specific advantages to boost the PCE. ${ }^{37-39}$ Among the reported metal oxides, $\mathrm{TiO}_{2}$ is an ETL material that has been most widely used by various research groups. However, Leijtens et al. reported lower stability of PSC devices with the use of $\mathrm{TiO}_{2}$ (due to its high photocatalytic activity under UV light) as an ETL material when compared with a $\mathrm{TiO}_{2}$-free PSC. ${ }^{40}$ Apart from the stability issue, another drawback of using $\mathrm{TiO}_{2}$ as an ETL material is that the film has to be annealed at a higher temperature, which not only can be a great barrier in the commercialization of PSC technology but is incompatible with the use of flexible substrates that may not bear such high temperatures. While the use of mesoporous $\mathrm{TiO}_{2}$ can achieve good efficiency, their counterpart, i.e. the planar PSC devices, suffers from hysteresis issues. A number of research groups have replaced $\mathrm{TiO}_{2}$ with $\mathrm{SnO}_{2}$ to achieve efficient, stable, and low-temperature processed PSCs. ${ }^{41}$ Researchers have also employed atomic layer deposited (ALD) $\mathrm{SnO}_{2}$, but the ALD process ${ }^{42}$ may not be as commercially feasible or cost-effective. Therefore, it is discernible that the commonly used metal oxides as ETLs have several drawbacks that need to be overcome. One of the strategies is the design and development of organic materials, and research over the past two years has helped to narrow the performance gap between the ETLs based on metal oxides and organic materials, thus, yielding a new generation of highly-efficient ETL materials. ${ }^{43,44}$ The key advantages of using organic materials include lower processing cost and straightforward synthetic protocols, higher solubility, and tunable optoelectronic properties through suitable molecular engineering. The majority of these organic materials feature a series of common design elements, e.g. are donor-acceptor (D-A) systems where an electron donating building block is linked to an electron accepting group through a $\pi$-linker. Another promising molecular design is an $\mathrm{A}-\mathrm{A}-\mathrm{A}$ format where a variety of acceptor building blocks can be joined to generate a target chromophore. Such systems are highly flexible and can be tuned according to the requirements of film transparency, solubility and chemical stability. Moreover, small 


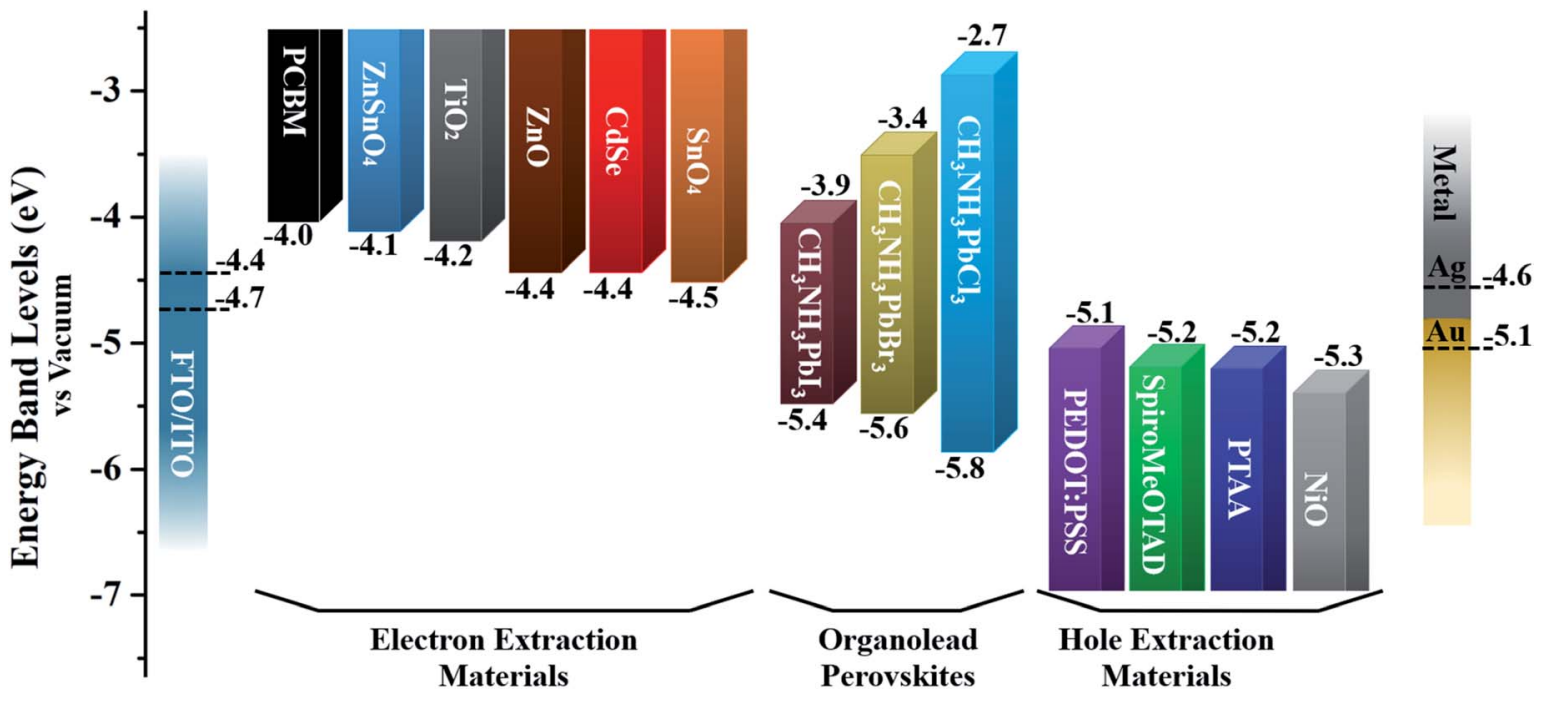

Fig. 5 The band energy level diagram for commonly utilized charge-extraction layer materials and $\mathrm{CH}_{3} \mathrm{NH}_{3} \mathrm{PbX}_{3}$ perovskites. Note: the dotted lines represent the work functions of various electrodes.

variations within these different sections, i.e. in $\mathrm{D}$ and $\mathrm{A}$, cause significant difference in photovoltaic performance. Recent literature suggests that the use and choice of an electron acceptor functionality plays a vital role in designing new targets - either small molecule or polymer based - as the developed target should possess desirable properties such as high electron mobility and excellent accepting strength. ${ }^{45-47}$ Therefore, it is important to extend research into organic materials to evaluate design strategies capable of building upon the merits described above. As depicted in Fig. 4, every PSC architecture uses selective interlayer materials, namely ETLs and hole transport layers (HTLs) which help to extract charges and improve PSC performance. A variety of inorganic and organic materials have been reported. For example, the most commonly used HTLs used in PSCs are Spiro-OMeTAD, ${ }^{48}$ PTAA, ${ }^{49}$ PEDOT:PSS ${ }^{50}$ and nickel oxide (NiO)..$^{51}$ Similarly, a variety of materials have been used as ETLs, including metal halides and oxides, ${ }^{52-55}$ organic small molecules,${ }^{56}$ conjugated polymers ${ }^{57}$ and non-conjugated polymers. ${ }^{58}$ These layers are often sandwiched between the electrodes and the perovskite layer, and their main function is to facilitate efficient charge extraction while reducing carrier recombination. Fig. 5 illustrates commonly used chargeextraction layers. ${ }^{59}$ Some argue that organic small molecules are particularly attractive for use as ETLs as they: (1) possess well-defined chemical structures, (2) are easy to synthesize through simple and straightforward synthetic strategies, (3) hold monodisperse molecular weight, and (4) can be purified using convenient purification methods such as through simple column chromatography. ${ }^{60-62}$

To improve the PCE of PSC devices, it is critical to choose the right ETL material whose energy levels complement those of both the electrodes and the light absorbing perovskite layer. A variety of organic HTL materials have been investigated in detail, and a number of reviews on their evolution and performance have been written. ${ }^{63-65}$ Curiously, even though ETL materials are considered to have a critical effect on the performance of PSCs, the development of organic ETL materials has lagged the development of HTL materials. To design an efficient organic ETL material, various parameters must be considered:

(1) The material should be highly soluble in an orthogonal solvent - a solvent that is different than the solvent of perovskite film fabrication - because the PSC device is a multilayer structure.

(2) The ETL material should possess good electron mobility and a common way to achieve this is through a conjugated structure. This is vital to improve the ability of electron transport to the cathode and deliver low series resistance.

(3) The film of an ideal ETL should easily coat the surface of the absorbing layer to avoid a direct contact between the electrode and perovskite layer, which could result in current leakage through local shunting.

(4) The energy levels, i.e. the LUMO and the HOMO, of an ETL molecule must complement the conduction and valence bands of the perovskite layer, respectively. Having said that, the LUMO of an ETL material should be lower than the conduction band and the HOMO value should be higher than the valence band of the perovskite layer, respectively. This is to mitigate the open-circuit voltage $\left(V_{\mathrm{oc}}\right)$ loss and to enhance electron extraction for optimum performance.

(5) An efficient ETL material should have electron rich centres, e.g. units containing heteroatoms such as nitrogen and sulfur. These units can act as Lewis bases and can coordinate with available $\mathrm{Pb}$ ions exposed at the surface to effectively passivate defects in the perovskite film. This could help to improve $V_{\text {oc }}$ and fill factor, hence, overall device performance.

(6) To complement the requirement of a material's dissolution in several orthogonal solvents, its printability must be considered in order to prevent the damage of the perovskite layer after spinning.

ETL materials can play a crucial role in the design of PSC device architecture and should be thought of as the backbone of 

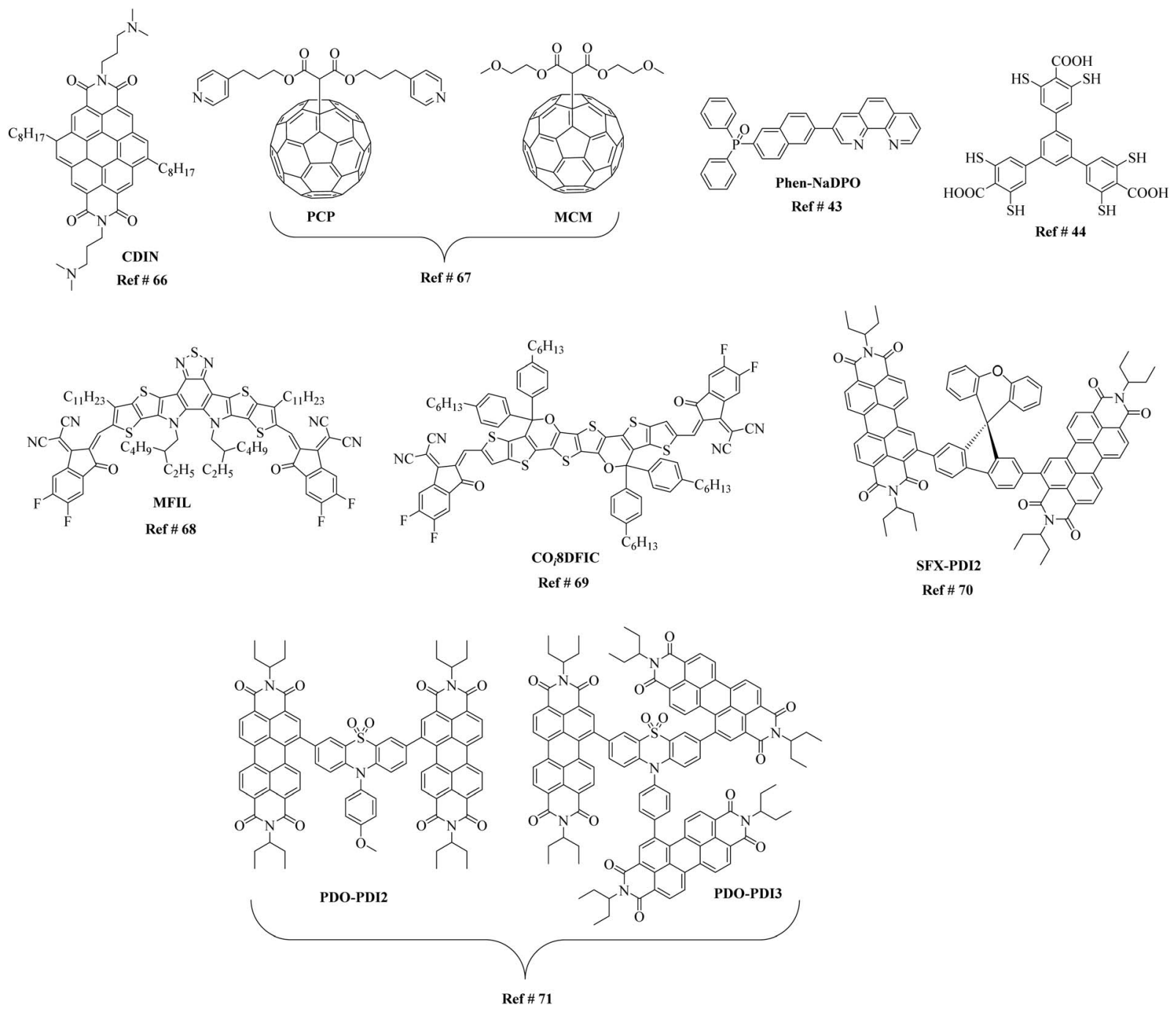

Fig. 6 Structural diversity in organic ETL materials of both fullerene and non-fullerene types.

this emerging technology. Therefore, the discovery of new materials that not only improve device performance but are based on straightforward synthetic strategies are critical. The beauty of organic-based materials is the structural diversity possible (Fig. 6), which lends itself to broad interpretability and optimisation of points 1-6 above. For example, an ETL material based on planar coronene and fabricated in a conventional $n-i-$ p PSC architecture reported a high efficiency of $>17 \%$ on a rigid and $14.2 \%$ on flexible devices. ${ }^{66}$ The fullerenes, di(3-(pyridin-4yl)propyl)[60]fullerenyl malonate (PCP) and di(1-methoxyethyl) [60]fullerenyl malonate (MCM), possess almost identical optoelectronic properties as ETL materials, affording efficiencies beyond $19 \%$ with a planar absorber layer over 1 micrometre, yet display different passivation strength influence on the hysteresis of the device through the change in terminal group. ${ }^{67}$ The two ETL materials, 3-[6-(diphenylphosphinyl)-2-naphthalenyl]1,10-phenanthroline (Phen-NaDPO) and its blend with tin(II) thiocyanate, i.e. Phen-NaDPO:Sn(SCN $)_{2}$, exhibited an improvement in the PCE from $16.6 \%$ to $18.2 \%$ in an inverted device configuration $\left((\mathrm{p}-\mathrm{i}-\mathrm{n}) ; \quad \mathrm{ITO} / \mathrm{NiO} / \mathrm{CH}_{3} \mathrm{NH}_{3} \mathrm{PbI}_{3} / \mathrm{PC}_{60} \mathrm{BM} / \mathrm{ETL} /\right.$
$\mathrm{Ag}) .{ }^{43}$ To show the structural diversity even more pronouncedly, thiol-functionalised 2D metal-organic framework built into a stable PSC device recorded a PCE of $22.0 \%$ along with substantially improved long-term operational stability. ${ }^{\mathbf{4 4}}$ Similarly, a multifunctional interfacial layer comprising a nonfullerene electron acceptor, Y6 (Fig. 6), was recently used to replace the conventionally used $\mathrm{PC}_{61} \mathrm{BM}$ and to enhance the device efficiency together with the operational stability of $\mathrm{p}-\mathrm{i}-\mathrm{n}$ structured devices. ${ }^{68}$ The presence of various functional groups on Y6, such as fluoro, cyano and sulfur, helped to increase the efficiency of electron transport, suppress ion migration to improve device performance, trap passivation, and enhance near-infrared photocurrent. Though the device geometry constructed was complex, a PCE of $21.0 \%$ was achieved, with enhanced operational stability (up to $1700 \mathrm{~h}$ ) upon exposure to light, heat, and moisture. Another example of a promising nonfullerene acceptor that has been used as an electron transport material is COi8DFIC (Fig. 6), which when combined with the perovskite, $\mathrm{MAPbBr}_{3}$, leads to high-performance, visible/NIR dual-colour photodetector with a structure of ITO/PTAA/ 
$\mathrm{MAPbBr}_{3} / \mathrm{CO} i 8 \mathrm{DFIC} / \mathrm{Ag} .{ }^{69}$ The new acceptor is an A-D-A type non-fullerene acceptor with an electron-rich carbon-oxygenbridged core unit and two electron-deficient terminal units. The acceptor affords high charge carrier mobility, low-energy light-harvesting, and a non-overlapping absorption with $\mathrm{MAPbBr}_{3}$. Even though the perovskite based on the combination of $\mathrm{MA}$ and $\mathrm{PbI}_{3}$ is often used, reports of a PSC with $\mathrm{CsPbI}_{2} \mathrm{Br}$ as a light absorption material and a binary ETL comprising $\mathrm{PC}_{61} \mathrm{BM}$ and SFX-PDI2 have been reported. ${ }^{70}$ The newly designed non-fullerene acceptor, SFX-PDI2, based on spiro[fluorene-9, $9^{\prime}$-xanthene] and perylene diimide structural units, were used in conjunction with the traditional acceptor, $\mathrm{PC}_{61} \mathrm{BM}$, to improve charge extraction, tune morphology. As a result, the binary ETL-based PSC afforded an efficiency of $15.12 \%$, a result that is among the highest efficiency numbers for inverted planar structured all-inorganic PSCs. Given the excellent photoelectron chemical properties and suitable energy level alignment with perovskite, perylene diimide-based ETL materials where the perylene diimide units were attached to a phenothiazine 5,5-dioxide (PDO) core building block to generate a dimer (PDO-PDI2) and a trimer (PDO-PDI3) have been reported. ${ }^{71}$ When applied in PSCs, the PDO-PDI3-based PSC exhibited an efficiency of $18.72 \%$ with no hysteresis and excellent long-term stability.

\section{NDI-based electron transport layer materials}

The use of functionalities such as naphthalene diimide (NDI, Fig. 7) in the design of next-generation ETL materials should be anticipated. The inclusion of NDI functionality in a given target is advantageous as not only is it a strong acceptor group and strong chromophore with appropriate physical and electronic properties to be considered for ETL materials, but the functional diversity helps to improve solubility of a given target by allowing substitution at its imide and/or core positions. ${ }^{72-78}$ The incorporation of a variety of solubilising chains, e.g. lipophilic, hydrophilic, alkoxy, aliphatic with terminal thiol groups, and polyethylene glycol, at imide positions enhances the solubility of a proposed target, a result that is of great significance to generate excellent pristine and blend films of the proposed target material without crystallisation. ${ }^{79-82}$ Therefore, it is timely to review the literature of ETL materials based on NDI functionality and to correlate the structural features with the device

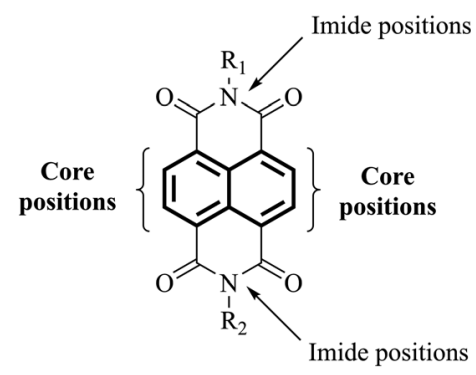

Fig. 7 Structure of naphthalene diimide (NDI) core. performance in order to determine useful design concepts to advance the area. This review provides the current state-of-theart in the field of ETL materials, both polymers and small molecules, comprising NDI functionality for PSCs and describes structure-property relationships. We also outline future perspectives from a materials chemistry viewpoint, outlining the correlation between structural aspects and device performance. It is critical to mention here that a number of factors, including environmental factors impacting stability of PSC devices and their toxicity and shorter lifetime, are already discussed in a number of recent review articles, ${ }^{\mathbf{8 3 - 9 0}}$ and the reader is encouraged to consider the respective sections of these reports for articles relevant to these specific areas. This review is written from a perspective of organic chemistry, and we believe this will provide fundamental advice on the future design of new ETL materials based on NDI format that will afford more efficient and stable PSCs.

The choice of an acceptor functionality is critical to design new ETL materials and that most of the high-performing organic ETL materials are conjugated structures, either through the merger of a variety of building blocks, for instance donor (D) and acceptor (A), or through the possession of a rigid, extended fused-ring backbone, and carry strong acceptor functionalities. Given the challenging structural requirements, for example presence of heteroatoms that can bind to the metal ions of the perovskite layer, and polar groups that can help lower the work function of cathodes, it is apparent that ETL materials based on NDI core should be at the forefront of efficient and logical material design. Briefly, an NDI unit is an electron deficient unit that possesses excellent photo, chemical, and thermal stabilities at room temperature, thus, making is suitable for applications such as light-harvesting, self-assembly, and electronic devices. ${ }^{91,92}$ A variety of electronic molecules based on NDI core have been prepared into supramolecular arrangements and have influenced the self-assembly of several other organic semiconductors, for example based on cyanopyridone and barbituric acid. ${ }^{\mathbf{9 3}, 94}$ The existence of electronwithdrawing imide fragments within the NDI permits them to be simply reduced chemically or electrochemically into stable anionic radicals while remaining stable to oxidation. Due to the latter, NDI acts as a stable n-type semiconducting core. It is arguable that NDI core offers unique advantages that cannot be considered universal with other functionalities such as fullerene and other non-fullerenes. For instance, the NDI unit is an electron poor unit that accommodates the LUMO density so precisely that it is easy to expand its electronic character using simple synthetic protocols. On the contrary, other non-fullerene acceptor functionalities such as rhodanine, cyanopyridone, and malononitrile do not possess this feature, thus, restricting their use for the development of ETL materials. Moreover, due to its additional properties, such as structural functionalisation at imide and core positions, and excellent light-absorbing in the high-energy region, NDI is known as a promising candidate for generating targets that can be used in applications such as OPVs, OFETs, and OLEDs. ${ }^{95-101}$ The same can be said for ETL materials for PSC technology. ${ }^{\mathbf{1 0 2}}$ 
NDIs generated through imide nitrogen substitution usually possess similar optoelectronic properties because the electron density nodes in the LUMO and the HOMO levels at imide positions reduce the coupling between the NDI unit and the imide substituents to a minimum. For typical NDIs, this leads to an optical transparency above $340 \mathrm{~nm}$. Having said that, this is an excellent way to functionalise NDI using a variety of connectors and to tune its processability. The targets that are generated using core substitutions are usually highly conjugated materials and depending upon the substitution, several parameters, such as solubility, optical bandgap, crystallinity, thermal and chemical stabilities, and light-harvesting, can be significantly altered. Moreover, such substitutions can assist in minimising the $\pi-\pi$ overlap between aggregating naphthalene subunits to improve the performance of the resulting devices by changing the morphology and self-assembly of NDIs. Furthermore, materials based on NDI format can be engineered to address the trade-off between device stability and efficiency. Such materials can be easily synthesized, scaled-up, and compete with champion ETL materials, for example fullerenes and metal oxides, by keeping desirable properties such as high electron mobility, solubility, chemical and thermal stabilities, and excellent accepting strength. Moreover, the use of both small molecule and polymeric approaches exists. Such advantages are likely to be responsible for prompting interest in the design of target ETL materials based on NDI functionality.

\subsection{Polymeric electron transport layer materials based on NDI format}

One of the first NDI-based ETL materials reported was polymeric. ${ }^{103}$ Three polymers, namely N2200/(P(NDI2OD-T2), PNVT8 , and P(NDI2OD-TT) (Fig. 8) were based on the conjunction of NDI and thiophene functionalities where the latter was arranged either linearly or in fused form. The incorporation of vinyl linkages into the polymer backbones maintained the LUMO energy levels, thereby facilitating electron injection. Inverted PSC devices were fabricated (device structure: ITO/ PEDOT:PSS $\left./ \mathrm{CH}_{3} \mathrm{NH}_{3} \mathrm{PbI}_{3-x} \mathrm{Cl}_{x} / \mathrm{ETL} / \mathrm{ZnO} / \mathrm{Al}\right)$ and the reported PCEs were $8.15 \%, 7.47 \%$, and $6.47 \%$, for N2200, PNVT-8 and $\mathrm{P}(\mathrm{NDI} 2 \mathrm{OD}-\mathrm{TT})$, respectively. The PCE results were competitive to the efficiency achieved using PCBM as the conventional ETL material of $8.51 \%$. For the higher-performing polymer (N2200), the thicknesses of the ETL and perovskite layers were $\sim 80 \mathrm{~nm}$ and $170 \mathrm{~nm}$, respectively. This work demonstrated that conjugated polymeric materials comprising $\mathrm{D}$ and A building blocks were efficient ETL materials and worth further exploration. Taking into account this combination and the success of $\mathrm{D}$ and A units, further reports emerged, for example an aminofunctionalized copolymer, PFN-2TNDI, where the polymeric backbone was composed of fluorene (D unit), naphthalene diimide (A unit), and thiophenes ( $\pi$-spacers) (Fig. 8). ${ }^{\mathbf{1 0 4}}$ The polymer exhibited good electron mobility of $4.8 \times 10^{-4} \mathrm{~cm}^{2} \mathrm{~V}^{-1}$ $\mathrm{s}^{-1}$ and was adequately soluble in a number of solvents to produce useful devices. This research realised that the presence of tertiary amine groups on the polymer side chain can passivate the surface traps of the perovskite layer to improve the electron extraction properties, which is now a growing area of research $^{\mathbf{1 0 5}}$ and can reduce the work function of the metal cathode by forming desired interfacial dipoles. The PSCs were fabricated with an inverted device architecture, ITO/ PEDOT:PSS/CH $\mathrm{CH}_{3} \mathrm{NH}_{3} \mathrm{PbI}_{3-x} \mathrm{Cl}_{x} / \mathrm{PFN}-2 \mathrm{TNDI} / \mathrm{Ag}$ ), and a high PCE of $16.7 \%$ was recorded - significantly higher than the efficiency obtained using the conventional ETL material PCBM (12.9\%). The optimum thickness of the electron extraction layer was found to be $50-100 \mathrm{~nm}$, which yielded an average PCE over 15\%. The devices based on this new polymer were found to be more stable when compared with the devices based on PCBM as the PCE dropped to $50 \%$ of its original value after 160 hours of storage time, while it only took 40 hours for PCBM-based devices to see a comparable reduction. The stability of devices was further attributed to the stronger packing between polymer chains, which provided a better physical barrier to reduce the water penetration rate to the perovskite layer - a major degradation source for PSCs.

Given the success of conjugated polymers as efficient ETL materials, new reports on a series of conjugated polymers, $\mathrm{P}(\mathrm{NDI} 2 \mathrm{OD}-\mathrm{T} 2), \mathrm{P}(\mathrm{NDI} 2 \mathrm{DT}-\mathrm{T} 2)$, and $\mathrm{P}(\mathrm{NDI} 2 \mathrm{OD}-\mathrm{TET})$ (Fig. 8), comparing the performance of the literature reported $\mathrm{P}(\mathrm{NDI} 2 \mathrm{OD}-\mathrm{T} 2)$ polymer with their structural analogues were made. ${ }^{\mathbf{1 0 6}}$ The longer side chains in these new polymers helped to enhance solubility, and hence processability, without influencing the energy levels and optical bandgap. The ethylene unit in the backbone of $\mathrm{P}(\mathrm{NDI} 2 \mathrm{OD}-\mathrm{TET})$ reduced the conjugation length, leading to a wider bandgap. Coating of ETL layers over perovskite film produced a much smoother surface, featuring a surface roughness of $\sim 4-6 \mathrm{~nm}$. The inverted devices were fabricated (ITO/PEDOT:PSS/ $\mathrm{CH}_{3} \mathrm{NH}_{3} \mathrm{PbI}_{3-x} \mathrm{Cl}_{x} / \mathrm{ETL} / \mathrm{Al}$ ) and exhibited PCEs of the order $10.82 \%, 10.83 \%$, and $0.18 \%$ for $\mathrm{P}(\mathrm{NDI} 2 \mathrm{OD}-\mathrm{T} 2), \mathrm{P}(\mathrm{NDI} 2 \mathrm{DT}-\mathrm{T} 2)$, and $\mathrm{P}(\mathrm{NDI} 2 \mathrm{OD}-\mathrm{TET})$, respectively. The maximum efficiency of the fabricated devices using the n-type polymers did not exceed the performance of devices based on the conventional PCBM ( $11 \%)$, though the authors claimed that the ambient stability of devices was considerably improved with the use of ETL polymers when compared with the device stability based on the latter. Given the success of (P(NDI2OD-T2)), as an efficient, n-type polymeric ETL material, together with its commercial availability, many research groups have now studied this polymer along with its structural analogues and by altering device fabrication conditions. In one such study, the electronic properties of N2200 were modified with a polymeric additive, poly[9,9-bis $\left(6^{\prime}-(N, N\right.$-diethylamino $)$ propyl)-fluorene-alt-9,9-bis(3-ethyl(oxetane-3-ethyloxy)-hexyl) fluorene] (PFN-Ox). ${ }^{107}$ The inverted devices were fabricated (ITO/ $\mathrm{NiO} / \mathrm{MAPbI}_{3} / \mathrm{N} 2200: \mathrm{PFN}-\mathrm{Ox} / \mathrm{bis}-\mathrm{C}_{60} / \mathrm{Ag}$ ) and the device performance was enhanced to $16.8 \%$ with the use of N2200:PFN-Ox as an electron extraction layer. The use of the conventional PCBM as an ETL material provided $16.62 \%$ efficiency, although the device's ambient stability was seen as inferior to that of devices based on N2200:PFN-Ox. This study demonstrated the potential of using polymeric ETLs to replace the commonly used PCBM to enhance the performance and stability of PSCs.

Efficient doping to high-performing polymers leads, in principle, to better ETL materials. For example, when PNDI-2T 


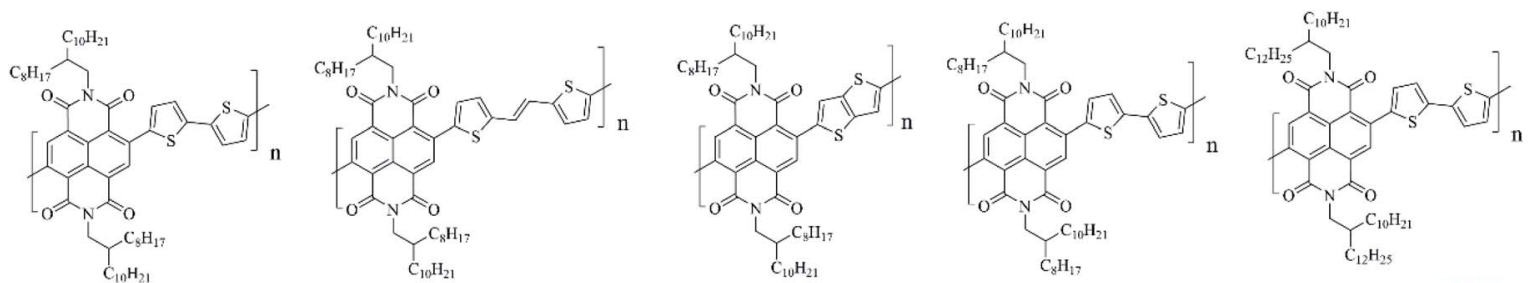

N2200 OR P(NDI2OD-T2) $(8.15 \%)$, I

PNVT-8 (7.47\%), I

P(NDI2OD-TT) (6.47\%), I

P(XDI2OD-T2) (10.82\%), I

P(NDI2DT-T2) (10.83\%), I
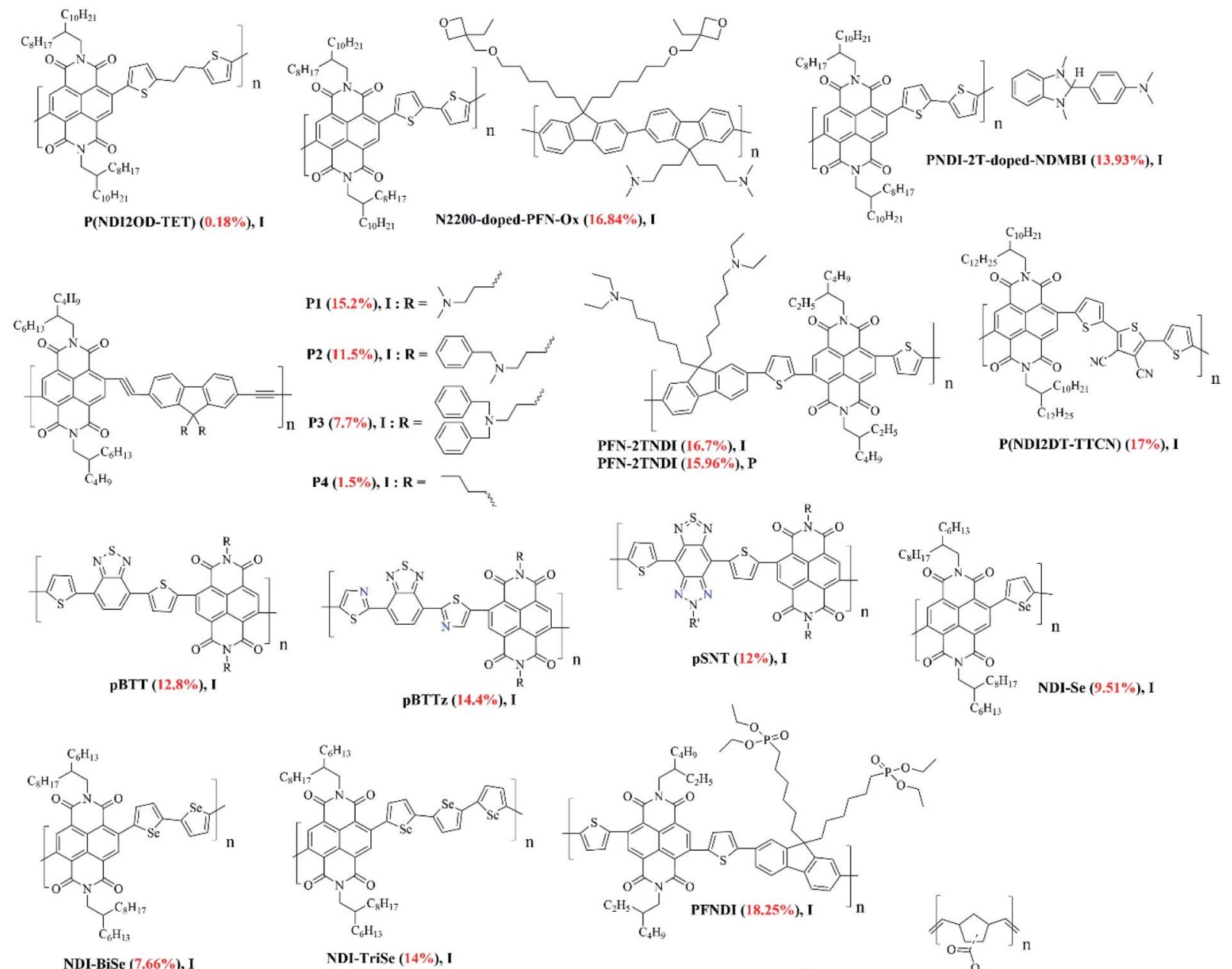

NDI-BiSe (7.66\%), I
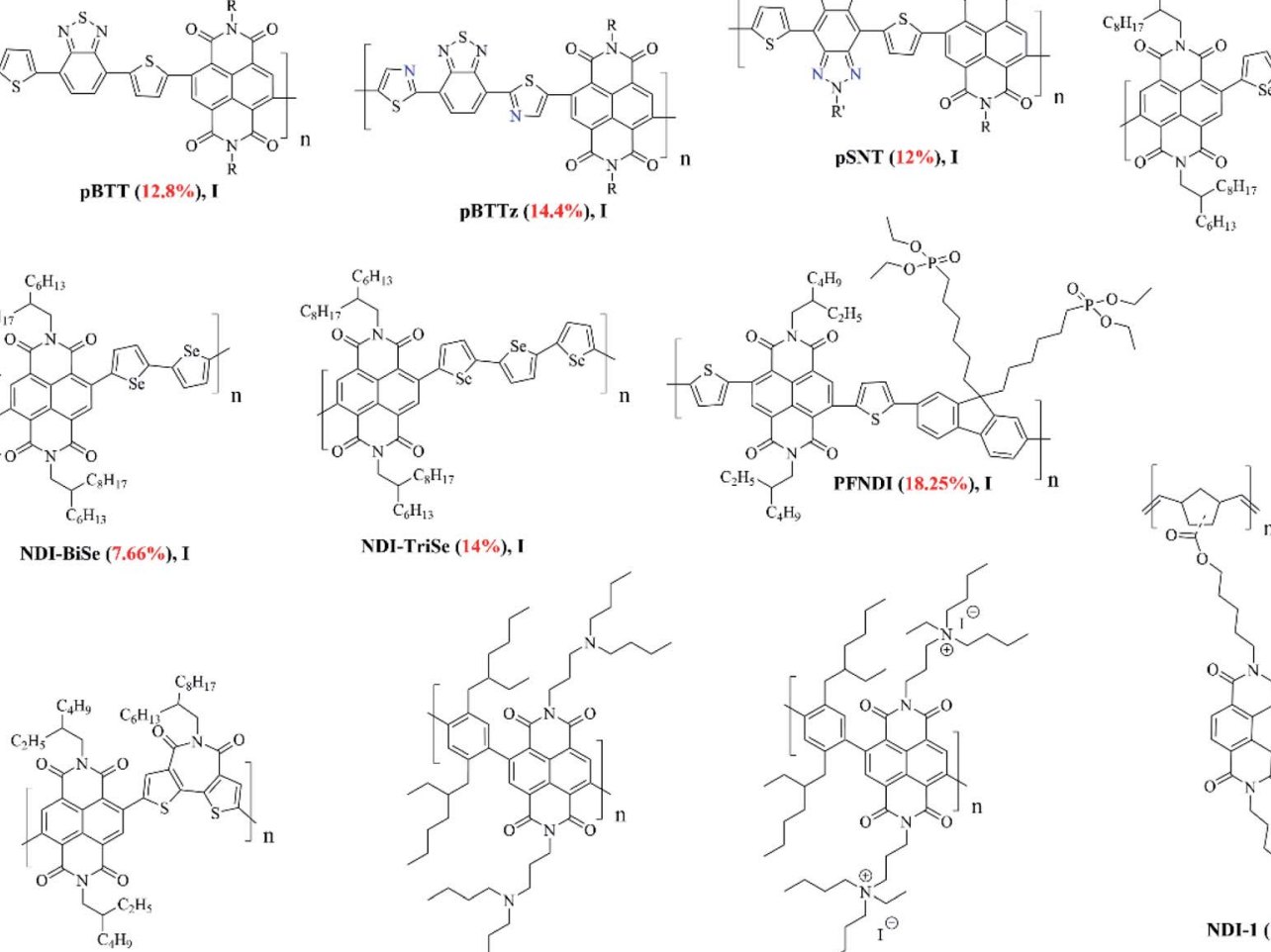

P(BTI-NDI) (19.5\%), I
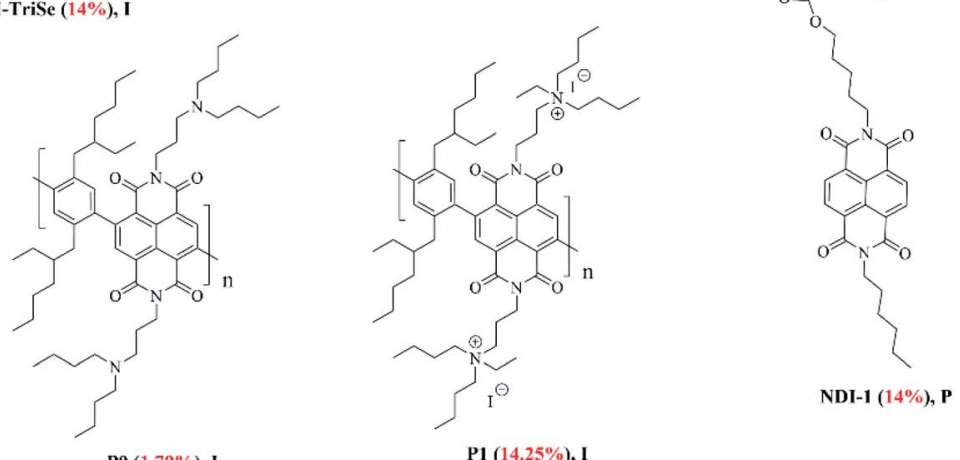

P0 (1.79\%), I

P1 (14.25\%), I

Fig. 8 The chemical structures of NDI-based polymers reported in this review article (I and P refer to inverted and planar devices). Optimal PCEs are quoted in brackets for each material.

is doped with (4-(1,3-dimethyl-2,3-dihydro-1H-benzoimidazol-2yl)phenyl)dimethylamine (N-DMBI) ${ }^{108}$ in fabricated inverted $\mathrm{p}^{-}$ i-n devices (glass/ITO/PEDOT:PSS/perovskite layer/ETL (PNDI-
2T/PNDI-2T + N-DMBI)/PEIE/Au), the doping doubled the efficiency from $6.5 \%$ to $13.93 \%$. Interestingly, this same study investigated the thickness of the electron extraction layer and 
found that a $20 \mathrm{~nm}$ thick film afforded the best device parameters. The film thickness of $50 \mathrm{~nm}, 35 \mathrm{~nm}$ and $14 \mathrm{~nm}$ provided PCEs of the order $3 \%, 12 \%$ and $\sim 10 \%$, respectively, thus, suggesting the importance of ETL thickness and its correlation with the device performance. Stability experiments indicated that the doped devices were far more stable than the undoped devices, where the former retained $85 \%$ of the PCE value after 150 hours and the latter kept $62 \%$ of the original value. ${ }^{108}$ The dopant, NDMBI, not only increases the conductivity of polymeric ETL, but also lowers the LUMO and the HOMO values, thus, improving both electron extraction and hole blocking abilities. Based on previous work, ${ }^{104}$ Li et al. reported the polymer PFN-2TNDI as an ETL material and demonstrated a $\mathbf{1 5 . 9 6 \%}$ efficiency in fabricated planar n-i-p devices (device architecture: ITO/PFN2TNDI/MAPbI ${ }_{3-x} \mathrm{Cl}_{x} /$ spiro-OMeTAD/Au), at optimized thickness of the ETL at $5 \mathrm{~nm}-$ a result which was comparable to that of inorganic $\mathrm{TiO}_{2}$ ETL-based devices (17.20\%). ${ }^{109}$ Moreover, the devices were processed at low temperature and showed enhanced photostability against UV irradiation when compared with the devices based on $\mathrm{TiO}_{2}$, thereby addressing, in part, photostability issues encountered in perovskite devices.

Polymer design can also be varied to achieve enhanced performance. A series of novel, n-type conjugated polymers, coded as P1 to P4, with amino-functionalized side groups were prepared (Fig. 8). ${ }^{\mathbf{1 1 0}}$ The copolymers comprised fluorene and NDI units within the identical backbone, but containing different pendants of $\mathrm{N}, \mathrm{N}$-dimethylamino, $\mathrm{N}$-methylbenzylamino, $N, N$-dibenzylamino, and pure alkyl chains. The choice of using NDI in the polymer backbone was based on the premise that it possesses large electron affinity and has been used to construct n-type organic semiconductors with excellent electron mobility for OPVs and OFETs. The amino functionalisation could be used to fine tune the work function of the cathode for better electron extraction and to passivate the surface traps of perovskite for a reduction of charge recombination, given the electron rich nitrogen heteroatom(s). The PSCs were fabricated based on the device configuration of ITO/ PEDOT:PSS $(\sim 40 \mathrm{~nm}) / \mathrm{MAPbI}_{3-x} \mathrm{Cl}_{x}(\sim 400 \mathrm{~nm}) / \mathrm{ETL}(\sim 50 \mathrm{~nm}) /$ $\mathrm{Ag}$, and the photovoltaic performance was studied. Although the authors reported two more polymers, P5 and P6, which were devoid of acetylene spacers, their fabrication into PSC devices was unreported. The PCEs of the PSC devices with P1 to P4 as the ETLs were $15.2 \%, 11.5 \%, 7.7 \%$, and $1.5 \%$, respectively. For the highest performing polymer P1, devices of various ETL thickness were fabricated. For an optimal thickness of $\sim 50 \mathrm{~nm}$, the devices based on a P1 interlayer produced a PCE over 15\%, however, as the thickness of interlayer increased past this, the PCE decreased. It was evident from this work that a number of structural elements are essential to create efficient ETL materials based on the NDI core. Structural features, such as (1) extended conjugation between NDI and donor units, (2) use of $\pi$-spacers, $e$.g. an acetylene unit, in a given molecular backbone, (3) inclusion of amino-functionalised groups, are critical and can help to generate an ETL target material with enhanced electron-transporting property. Moreover, with the inclusion of such structural features it is viable to develop novel thicknessinsensitive ETL materials, and this in many respects is proportional to electron mobility. Kim et al. reported the polymer (P(NDI2DT-TTCN)) composed of NDI and donor oligothiophenes with one of the thiophenes bearing electronwithdrawing dicyano functionalities as an ETL material (Fig. 8). ${ }^{111}$ Within inverted $\mathrm{p}-\mathrm{i}-\mathrm{n}$ devices (device architecture: glass/ITO/HTL/ $\mathrm{CH}_{3} \mathrm{NH}_{3} \mathrm{PbI}_{3} / \mathrm{ETL} /$ metal) with $\mathrm{P}(\mathrm{NDI} 2 \mathrm{DT}-\mathrm{TTCN})$ and PCBM as electron extraction layers, the performance of devices based on P(NDI2DT-TTCN) significantly outperformed the devices based on PCBM alone (17.0 vs. $14.3 \%)$. The enhanced performance was attributed to the polymer's ability to improve the electron extraction together with the long-term stability of PSC devices under ambient conditions. The P(NDI2DT-TTCN)-based electron-only device showed a higher electron mobility $\left(\mu_{\mathrm{e}}=7.71 \times 10^{-4} \mathrm{~cm}^{2} \mathrm{~V}^{-1} \mathrm{~s}^{-1}\right)$ when compared with the device based on PCBM $\left(2.53 \times 10^{-4} \mathrm{~cm}^{2} \mathrm{~V}^{-1}\right.$ $\mathrm{s}^{-1}$ ), suggesting that $\mathrm{P}$ (NDI2DT-TTCN) could promote a better vertical charge transfer than PCBM for the inverted PSC devices. This report provided some valuable design guidelines for originating new polymeric ETL materials for high performances in flexible PSCs.

Three conjugated polymers, pBTT, pBTTz, and pSNT, based on the donor-acceptor ${ }_{1}$-donor-acceptor ${ }_{2}\left(\mathrm{D}-\mathrm{A}_{1}-\mathrm{D}-\mathrm{A}_{2}\right)$ format were designed and synthesised as ETL materials for PSCs (Fig. 8). ${ }^{112}$ In addition, the effect of substitution position of $\mathrm{sp}^{2}-\mathrm{N}$ atoms on the PSC performance was investigated. From a structural perspective, the investigated polymers are NDIbased, n-type polymers, with field-effect electron mobilities up to $0.92,0.46$, and $4.87 \mathrm{~cm}^{2} \mathrm{~V}^{-1} \mathrm{~s}^{-1}$, respectively. Since they demonstrated good performance in organic and tandem solar cells, the authors of this research surmised they should perform well in PSC devices. The fabrication of pBTT, pBTTz, and pSNT as ETLs in inverted PSC devices (ITO/PEDOT:PSS/ $\mathrm{CH}_{3} \mathrm{NH}_{3} \mathrm{PbI}_{3-x} \mathrm{Cl}_{x} /$ ETLs/Ag) afforded PCEs of the order of $12.8 \%, 14.4 \%$, and $12.0 \%$, respectively. Though the introduction of $\mathrm{sp}^{2}-\mathrm{N}$ atoms into the donor thiophene units of pBTT (producing pBTTz) exhibited a slightly lower electron mobility $\left[\mu_{\mathrm{e}}(\mathrm{pBTT})=0.92 \mathrm{~cm}^{2} \mathrm{~V}^{-1} \mathrm{~s}^{-1} ; \mu_{\mathrm{e}}(\mathrm{pBTTz})=0.46 \mathrm{~cm}^{2}\right.$ $\left.\mathrm{V}^{-1} \mathrm{~s}^{-1}\right]$, the PCE of PSCs were still significantly improved. On the contrary, pSNT with two additional $\mathrm{sp}^{2}-\mathrm{N}$ atoms and very high electron mobility $\left[\mu_{\mathrm{e}}(\mathrm{pSNT})=4.87 \mathrm{~cm}^{2} \mathrm{~V}^{-1} \mathrm{~s}^{-1}\right]$ showed only moderate PSC performance. The electron-rich sulfur atoms (in donor thiophene units) appear to have a strong impact on the passivation of under-coordinated Pb-atoms, as reflected by the current density-voltage hysteresis curves of the pBTTz-based devices. Given the presence of electron-rich heteroatoms and NDI functionality, the polymers were hydrophobic in nature and were thus able to retain device stability over a substantial amount of time, e.g. the pBTTzbased devices retained $80 \%$ of its stability after ten days. This study was the first demonstration where the effect of $\mathrm{sp}^{2}$ $\mathrm{N}$ position in the main polymer chain, i.e. in the backbone of the parent polymer pBTT, on the photovoltaic properties of PSCs was investigated.

It is evident that the combination of NDI with donor functionalities, and thiophene in particular, produced polymer backbones that were rich in conjugation, offered excellent electron mobilities, exhibited adequate solubility in a variety 
of solvents, and were used as efficient ETL materials. In moving from sulfur to selenium, Yan produced three semiconducting polymers, NDI-Se, NDI-BiSe, and NDI-TriSe, via copolymerization with NDI for use as ETL materials (Fig. 8). ${ }^{113}$ The respective polymers utilized one, two and three selenophene units in conjunction with NDI in the main polymer chains. The three polymers demonstrated excellent thermal stability, high crystallinity, and energy levels complimentary to the perovskite absorber layer. The polymers were fabricated in an inverted device architecture with a configuration of ITO/ $\mathrm{NiO}_{x} / \mathrm{MAPbI}_{2.8} \mathrm{Cl}_{0.2} / \mathrm{ETL} / \mathrm{Ag}$, and the highest efficiency was achieved with the incorporation of NDI-TriSe $(14.0 \%)$, an efficiency comparable to the standard cell using thermally deposited $\mathrm{C}_{60} / \mathrm{BCP}$ bilayer ETL (14.6\%). The use of bis- and tris-selenophene units increased polymer planarity and delocalization, and thus conductivity $\left[\mu_{\mathrm{e}}(\mathrm{NDI}-\mathrm{TriSe})=3.0 \times 10^{-4}\right.$ $\mathrm{cm}^{2} \mathrm{~V}^{-1} \mathrm{~s}^{-1}$ vs. $\mu_{\mathrm{e}}($ NDI-BiSe $)=6.0 \times 10^{-5} \mathrm{~cm}^{2} \mathrm{~V}^{-1} \mathrm{~s}^{-1}$ and $\mu_{\mathrm{e}}$ $\left.(\mathrm{NDI}-\mathrm{Se})=4.3 \times 10^{-5} \mathrm{~cm}^{2} \mathrm{~V}^{-1} \mathrm{~s}^{-1}\right]$. Though the polymer decomposition temperature gradually decreased with the increase in selenophene units, the decomposition temperature of all three polymers was far beyond the frequently used annealing temperature for optoelectronic device applications (100-200 ${ }^{\circ} \mathrm{C}$ ). The thickness of the ETL was optimized to be only $\sim 9 \mathrm{~nm}$, which is considered thin relative to other ETLs used in PSC devices. Deng reported a novel NDI-based conjugated polymer PFNDI by introducing phosphite ester side chains (Fig. 8). ${ }^{114}$ As a result, PFNDI afforded good solubility in chlorobenzene, a solvent which is compatible with the solution processing of perovskite layers and the generation of smoother films. The introduction of the phosphite ester groups was helpful for the PFNDI to adhere to the perovskite and passivate defects. SEM analysis indicated that the perovskite layer surface was covered by PFNDI layer without any pinholes. From the AFM analysis, it was evident that the perovskite surface was much smoother after it was covered by PFNDI, with the surface root-mean-square roughness reduced from $7.27 \mathrm{~nm}$ to $5.09 \mathrm{~nm}$. An inverted device architecture (glass/ITO/HTL/perovskite/PFNDI/C 60 /BCP/Ag) as PSC devices were prepared with a thickness of $\sim 5 \mathrm{~nm}$. Devices prepared without PFNDI exhibited a PCE of $17.41 \%$, whereas for the PFNDI-based devices this improved to $18.25 \%$, realizing stable PSCs under atmospheric conditions.

Recently, Y. Shi et al. reported an A-A-based polymer, coded as P(BTI-NDI), based on NDI functionality (Fig. 8). ${ }^{115}$ The main rational of their design was to replace the conventional $\mathrm{PC}_{61} \mathrm{BM}$ and to improve the efficiency and stability of PSC devices. The developed polymer exhibited high thermal decomposition temperature of $405{ }^{\circ} \mathrm{C}$ and a reasonable electron mobility $(4.2 \times$ $10^{-4} \mathrm{~cm}^{2} \mathrm{~V}^{-1} \mathrm{~s}^{-1}$ ) that was deduced using the space-chargelimited current (SCLC) method. The PSCs were fabricated with an inverted device configuration of ITO/PTAA/perovskite/ETL/ $\mathrm{BCP} / \mathrm{Ag}$ to probe the performance of $\mathrm{P}(\mathrm{BTI}-\mathrm{NDI})$ and to compare with the conventionally used $\mathrm{PC}_{61} \mathrm{BM}$. The $\mathrm{P}(\mathrm{BTI}-\mathrm{NDI})-$ based device achieved remarkable PCE of 19.5\% with negligible hysteresis. Though the efficiency using $\mathrm{PC}_{61} \mathrm{BM}$ was $20.1 \%$, the authors claimed that the newly designed ETL material showed improved stability compared to $\mathrm{PC}_{61} \mathrm{BM}$-based devices. This was primarily attributed to the higher hydrophobicity of the new ETL. They also reported that a thickness of $69 \mathrm{~nm}$ was found to be optimal for the higher device outcome.

In other work, S. Valero et al. outlined three novel, NDIbased materials, namely P0, P1 and M1, bearing ternary or quaternary ammonium moieties (Fig. 8). ${ }^{\mathbf{1 1 6}}$ The main aim of designing these materials was to use them as buffer layers between fullerene and the metal cathode to improve the performance of PSCs. P1 was an ammonium-bearing NDI polymer, together with its ternary amine polymer counterpart, P0, and its small molecule analogue, M1 (note: for structures of P0 and P1, see Fig. 8 and for M1, see Fig. 9). All materials were found to be thermally stable as their thermal behaviour was investigated by means of thermogravimetric analysis (TGA) and differential scanning calorimetry (DSC) analyses. The newly developed materials demonstrated suitable energy levels with low-lying LUMOs around $4 \mathrm{eV}$ and the HOMOs around $6 \mathrm{eV}$. They were soluble in polar solvents, thus, allowing the authors to use them as buffer layers, with no risk of dissolving the underlying $\mathrm{PC}_{61} \mathrm{BM}$ layer during deposition. The chosen architecture of the devices was simple and inverted: FTO glass/ $\mathrm{NiO}_{x} / \mathrm{CH}_{3} \mathrm{NH}_{3} \mathrm{PbI}_{3} / \mathrm{PC}_{61} \mathrm{BM} /$ buffer layer/Au. They compared the new materials as buffer layers against the conventionally used buffer layer, bathocuproine (BCP), for $\mathrm{PC}_{61} \mathrm{BM}$-based devices. Devices were also fabricated with no buffer layer so that $\mathrm{PC}_{61} \mathrm{BM}$ directly contacts with the gold cathode. The device performances with polymer P1 and small molecule M1 were highly comparable to those obtained with the reference BCP buffer layer. BCP gave an average $11.82 \%$ PCE and both M1 and P1 gave average values of $11.42 \%$ and $11.43 \%$, respectively. Without any buffer layer, an average value of $3.48 \%$ was reported. It was established from this study that the halogen ammonium salts carry a great potential to improve the performance of perovskite devices and that NDI-based materials are very promising candidates to improve the performance and stability of PSCs.

A very recent report by K. A. Kurdi et al. mentions a number of NDI-based materials where a new side-chain polymer, NDI1 , is tested against other NDI small molecules as well as $\mathrm{SnO}_{2}$ as a reference (note: the structure of NDI-1 is given in Fig. 8 and of NDI-2, NDI-3 and NDI-4 in Fig. 9). ${ }^{117}$ This new sidechain polymer exhibited: (1) good transparency throughout the visible region, (2) adequate solubility in low- to mediumpolarity organic solvents, (3) resistance to dipolar aprotic solvents such as dimethylformamide, and (4) an appropriate electron affinity for use as an electron extraction layer in leadhalide PSCs. It was established by TGA and DSC that the new side-chain polymer, NDI-1, is thermally stable, with decomposition temperatures above $250^{\circ} \mathrm{C}$, together with other small molecule NDIs, indicating the compatibility with temperatures needed for subsequent deposition and annealing of many hybrid perovskites used in PSCs. The regular $n-i-p$ perovskite devices were fabricated and the performance of NDI-1 was compared to that of several small-molecule NDI derivatives and of $\mathrm{SnO}_{2}$. The best performing device using NDI-1 afforded $14.0 \%$ efficiency which was slightly lower than the efficiency achieved using $\mathrm{SnO}_{2}$ (15.6\%). Though the 

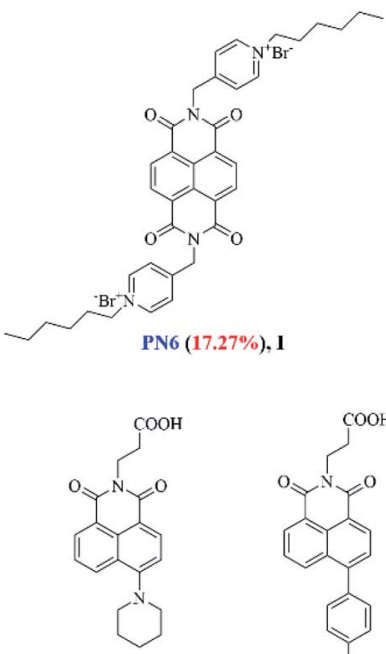

PN-P (5.45\%), P

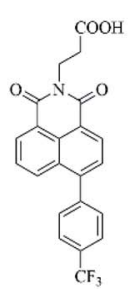

TN-P (5.41\%), P

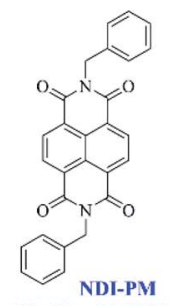

(18.4\% with $\mathrm{MAPbI}_{3}$ ), I $\left(19.6 \%\right.$ with $\left.\mathrm{FAPbI}_{3 \times x} \mathrm{Br}_{3}\right)$, I

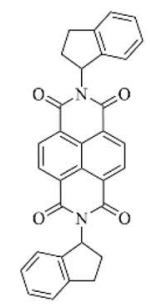

NDI-ID (20.2\%), I

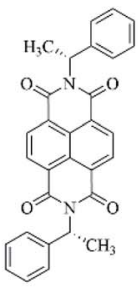

NDI-PhE $(20.5 \%)$, I

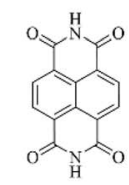

NDI-H (10.7\%), P
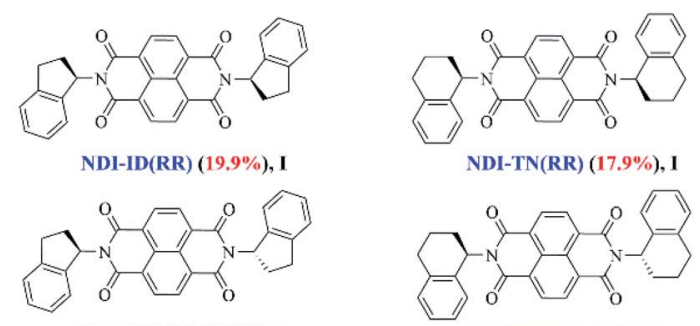

NDI-ID(RS) (19.4\%), I
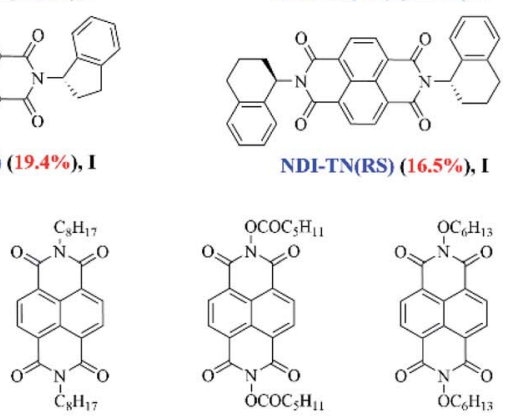

NDI-2 $(9.8 \%)$, P

NDI-3 (4.5\%), P NDI-4 (5.5\%), P

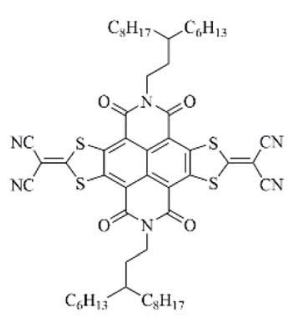

NDI3HU-DTYM2 (8.7\%), I
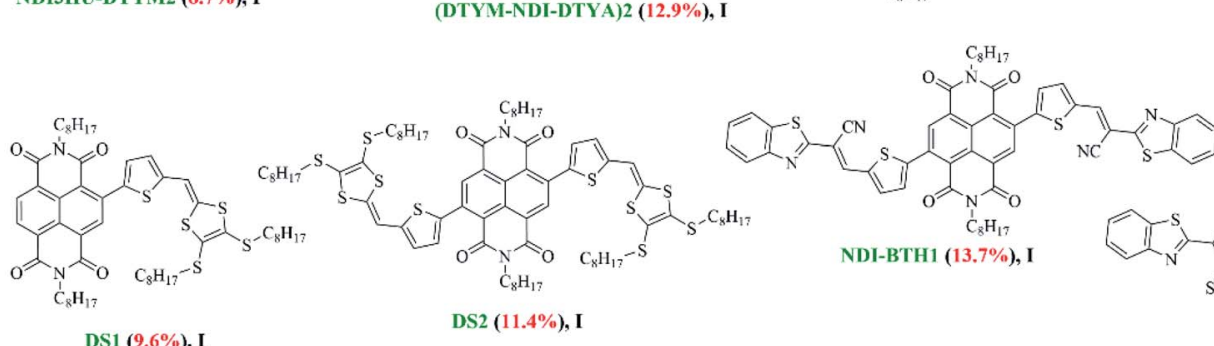

NDI-BTH1 $(13,7 \%)$, I
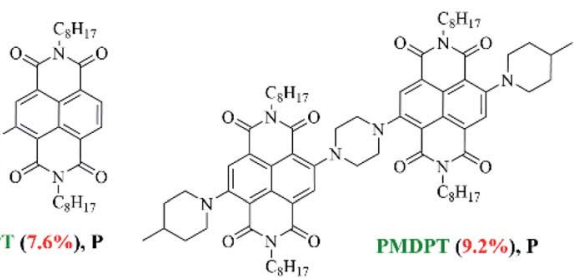

PDPT (7.6\%), P

$\mathrm{C}_{8} \mathrm{H}_{17}$

PMDPT $(9.2 \%)$, P

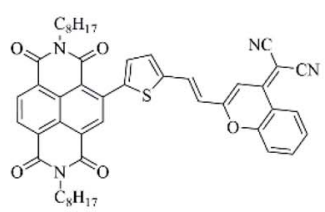

NDIF1 (15.6\%), I

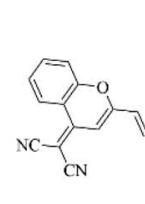

(1)

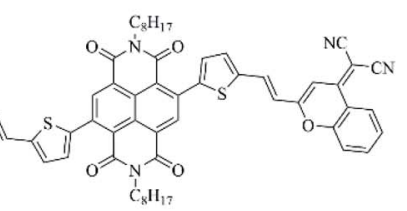

NDIF2 (11.1\%), I

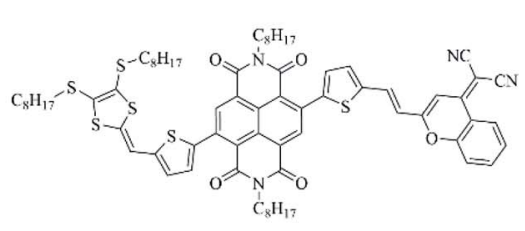

DNDIF3 (11.7\%), I

Fig. 9 The chemical structures of NDI-based small molecules reported in this review article (I and P refer to inverted and planar devices). Optimal PCEs are quoted in brackets for each material.

efficiency using NDI-1 was lower, it was claimed that the devices comprising the polymer exhibited greater stability than $\mathrm{SnO}_{2}$-based control devices when aged under the one sun conditions for almost 300 hours at $60{ }^{\circ} \mathrm{C}$. This study provided a foundation for designing new NDI derivatives that could be used as dopant-free, solution-processable ETL materials and 
could potentially replace the conventionally used, metal oxide ETLs such as $\mathrm{SnO}_{2}$.

The photovoltaic performance parameters for each of the polymer systems mentioned in this section are also tabulated in Table 1 to be used as a comparable guide.
3.2 Small molecule electron transport layer materials based on an NDI format

It is evident that highly conjugated polymers based on the NDI format have received significant attention as efficient ETL

Table 1 Photovoltaic performance parameters of polymeric NDI-based ETLs for PSCs

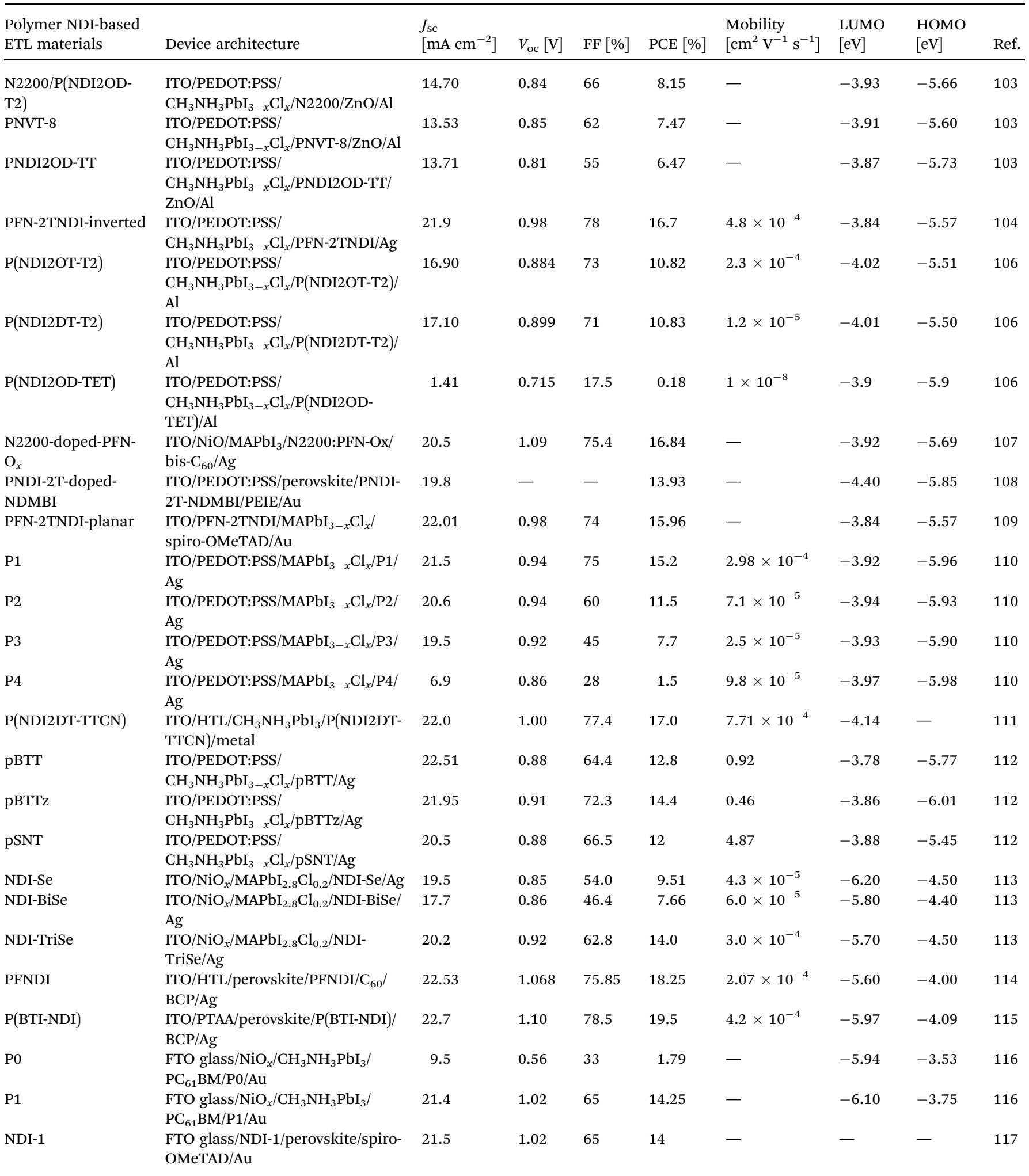


Table 2 Photovoltaic performance parameters of small molecule NDI-based ETLs for PSCs

\section{Small molecule NDI-}

based

ETL materials

ETL materials Device architecture

PN6

NDI-PM/MAPbI

\section{NDI-PM/}

$\mathrm{FAPbI}_{3-x} \mathrm{Br}_{x}$

NDI-ID

NDI-PhE

NDI-H

PN-P

TN-P

NDI-P

NDI-ID (RR)

NDI-TN (RR)

NDI-TN (RS)

NDI3HU-DTYM2

(DTYM-NDI-DTYA) $)_{2}$

PDPT

PMDPT

DS1

DS2

NDI-BTH1

NDI-BTH2

NDIF1

NDIF2

NDIF3

NDI-BN

M1

NDI-2

NDI-3

NDI-4

$\mathrm{ITO} / \mathrm{NiO}_{x} /$ perovskite/ $\mathrm{PC}_{61} \mathrm{BM} /$
$\mathrm{PN} 6 / \mathrm{Ag}$
PN6/Ag

ITO/PEDOT:PSS/MAPbI ${ }_{3} / \mathrm{NDI}-\mathrm{PM} / \quad 21.1$ Al

ITO/PEDOT:PSS/FAPbI ${ }_{3-x} \mathrm{Br}_{x} / \mathrm{NDI}-\quad 22.8$

$\mathrm{PM} / \mathrm{Al}$

ITO/PEDOT:PSS/FAPbI ${ }_{3-x} \mathrm{Br}_{x} / \mathrm{NDI}-\quad 23.0$

ID/Al

ITO/PEDOT:PSS/FAPbI ${ }_{3-x} \mathrm{Br}_{x} / \mathrm{NDI}-\quad 23.1$

$\mathrm{PhE} / \mathrm{Al}$

ITO/PEIE/NDI-H/MAPbI ${ }_{3} /$ spiro- $^{-} \quad 18.9$

OMeTAD/Au

ITO/PN-P/MAPbI ${ }_{3} /$ spiro-OMeTAD/ 10.23

$\mathrm{Au}$

ITO/TN-P/MAPbI $/$ /spiro-OMeTAD/ 10.93

$\mathrm{Au}$

ITO/NDI-P/MAPbI ${ }_{3} /$ spiro-

20.57

OMeTAD/Au

ITO/PEDOT:PSS/

$\left(\mathrm{FAPbI}_{3}\right)_{x}\left(\mathrm{MAPbBr}_{3}\right)_{1-x} / \mathrm{NDI}-\mathrm{ID}$

(RR)/Al

ITO/PEDOT:PSS/

$\left(\mathrm{FAPbI}_{3}\right)_{x}\left(\mathrm{MAPbBr}_{3}\right)_{1-x} / \mathrm{NDI}-\mathrm{ID}$

(RS)/Al

ITO/PEDOT:PSS/

$\left(\mathrm{FAPbI}_{3}\right)_{x}\left(\mathrm{MAPbBr}_{3}\right)_{1-x} / \mathrm{NDI}-\mathrm{TN}$

(RR)/Al

ITO/PEDOT:PSS/

$\left(\mathrm{FAPbI}_{3}\right)_{x}\left(\mathrm{MAPbBr}_{3}\right)_{1-x} / \mathrm{NDI}-\mathrm{TN}$

(RS)/Al

ITO/PEDOT:PSS/MAPbI ${ }_{3}$ /

NDI3HU-DTYM2/Ag

ITO/PEDOT:PSS/MAPbI ${ }_{3} /$ (DTYM-

NDI-DTYA) $)_{2} / \mathrm{Ag}$

ITO/PEDOT:PSS/

$\mathrm{CH}_{3} \mathrm{NH}_{3} \mathrm{PbI}_{3-x} \mathrm{Cl}_{x} / \mathrm{PDPT} / \mathrm{Ag}$

ITO/PEDOT:PSS/

$\mathrm{CH}_{3} \mathrm{NH}_{3} \mathrm{PbI}_{3-x} \mathrm{Cl}_{x} / \mathrm{PMDPT} / \mathrm{Ag}$

ITO/PEDOT:PSS/

$\mathrm{CH}_{3} \mathrm{NH}_{3} \mathrm{PbI}_{3-x} \mathrm{Cl}_{x} / \mathrm{DS} 1 / \mathrm{Ag}$

ITO/PEDOT:PSS/

$\mathrm{CH}_{3} \mathrm{NH}_{3} \mathrm{PbI}_{3-x} \mathrm{Cl}_{x} / \mathrm{DS} 2 / \mathrm{Ag}$

ITO/PEDOT:PSS/

$\mathrm{FA}_{y} \mathrm{MA}_{1-y} \mathrm{PbI}_{3-x} \mathrm{Cl}_{x} / \mathrm{NDI}-\mathrm{BTH} 1 / \mathrm{Ag}$

ITO/PEDOT:PSS/

$\mathrm{FA}_{y} \mathrm{MA}_{1-y} \mathrm{PbI}_{3-x} \mathrm{Cl}_{x} / \mathrm{NDI}-\mathrm{BTH} 2 / \mathrm{Ag}$

ITO/PEDOT:PSS/perovskite/

NDIF1/Ag

ITO/PEDOT:PSS/perovskite/

NDIF2/Ag

ITO/PEDOT:PSS/perovskite/

NDIF3/Ag

ITO/PTAA/perovskite/ $\mathrm{PC}_{61} \mathrm{BM} /$

NDI-BN/Ag

FTO glass $/ \mathrm{NiO}_{x} / \mathrm{CH}_{3} \mathrm{NH}_{3} \mathrm{PbI}_{3} /$

$\mathrm{PC}_{61} \mathrm{BM} / \mathrm{M} 1 / \mathrm{Au}$

FTO glass/NDI-2/perovskite/spiroOMeTAD/Au

FTO glass/NDI-3/perovskite/spiro- 11.6

OMeTAD/Au

FTO glass/NDI-4/perovskite/spiro-

OMeTAD/Au

20.53

22.9

22.7

21.9

21.5

18.56

22.80

22.9

22.4

23.41

22.65

21.3

22.13

23.66

18.91

22.11

23.61

21.8

18.3

14.8
$J_{\mathrm{sc}} \quad$ Mobility LUMO HOMO

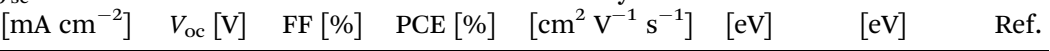

$1.11 \quad 76.07 \quad 17.27$

$7.1 \times 10^{-5}$

$-4.07$

$-7.02$

118

$\begin{array}{llll}1.1 & 79.1 & 18.4 & 1.7 / 0.72\end{array}$

119

$\begin{array}{llll}1.08 & 79.6 & 19.6 & 1.7 / 0.72\end{array}$

$-$

- 119

$1.1 \quad 80$

20.2

$1.5 \times 10^{-5}$

- $\quad 120$

$1.1 \quad 80.8$

20.5

$1.5 \times 10^{-5}$

-

$-$

121

$\begin{array}{lllllll}0.88 & 64 & 10.7 & 5.1 \times 10^{-5} & -4.0 & -6.9 & 122\end{array}$

$0.76 \quad 69.69$

5.45

$-$

$-3.01$

$-5.59$

123

$0.94 \quad 52.94 \quad 5.41 \quad-$

$\begin{array}{lll}-3.14 & 6.37 & 123\end{array}$

$1.05 \quad 73.98 \quad 16.01$

$-3.83$

6.96

123

$1.1 \quad 79.1$

19.9

$3.5 \times 10^{-4}$

-

$-$

125

1.0978

19.4

$5.5 \times 10^{-5}$

125

$1.06 \quad 77$

17.9

$4.4 \times 10^{-6}$

125

$1.05 \quad 73.1$

16.5

$8.6 \times 10^{-6}$

125

$0.96 \quad 48.85$

8.7

$7.50 \times 10^{-4}$

$-4.38$

$-6.38$

127

$0.9 \quad 62.8$

12.9

$6.26 \times 10^{-3}$

$-4.25$

$0.76 \quad 44$

7.6

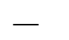

$-3.73$

$-5.65$

127

$0.84 \quad 49$

9.2

-

$\begin{array}{lll}-3.65 & -5.48 & 128\end{array}$

$0.74 \quad 55.4$

9.6

-

$\begin{array}{lll}-4.2 & -5.41 & 129\end{array}$

$0.8 \quad 63$

11.4

$-$

$0.96 \quad 67$

13.7

$14 \times 10^{-5}$

$-5.34$

129

$0.98 \quad 71$

15.4

$8.64 \times 10^{-4}$

$-4.32$

$-5.97 \quad 130$

$97 \quad 64$

15.6

$5.91 \times 10^{-6}$

$-4.24$

$-5.78 \quad 130$

$80 \quad 68.8$

11.1

$1.53 \times 10^{-5}$

$-3.8$

$-5.68$

131

$91 \quad 56$

11.7

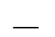

$-5.60$

131

21.32

$1.0 \times 10^{-6}$

$-3.8$

$-5.00 \quad 131$

$1.04 \quad 60$

13.73

$-$

$0.98 \quad 56$

9.8

$0.91 \quad 43$

4.5

-

5.5

$0.84 \quad 46$ 
materials in the field of PSCs, and rightly so, achieving impressive efficiencies for organic materials of over $18 \%$. Although an encouraging outcome, there are a reported number of disadvantages associated with their use such as ability to purify, batch-to-batch variations in terms of molecular weight and purity, their lower versatility for deposition processes, e.g., they cannot be deposited via thermal evaporation, and end group deviation. These are all critical considerations and can compromise the overall device outcome. In contrast, small molecules overcome many of these disadvantages, on top of straightforward synthetic protocols and structural modifications to tune the optical bandgap. As a result, small molecules have recently made significant inroads into the PSC research area. Importantly, we demonstrate the flexibility that small molecules based on NDI can be designed by substitutions at either imide nitrogen atoms (axial position) or core positions (Fig. 7). The current literature suggests that both categories can produce efficient ETL materials for PSCs (vide infra). These are summarised in Table 2.

3.2.1 Axially-substituted NDI derivatives. One of the first reported compounds for PSC utilisation was a wide bandgap, water/alcohol-soluble small molecule, labelled PN6, as a dicationic compound based on $\mathrm{N}, \mathrm{N}$-difunctionalised NDIs bearing pyridinium salts (Fig. 9). ${ }^{\mathbf{1 1 8}}$ The axial design considered was based on the premise that NDI derivatives based on such substitutions offer excellent electron mobility, adequate solubility, high chemical stability, and, above all, can be synthesised using simple synthetic strategies. PN6 exhibited a large optical bandgap $(2.95 \mathrm{eV})$, high electron mobility $\left(7.1 \times 10^{-5} \mathrm{~cm}^{2} \mathrm{~V}^{-1}\right.$ $\left.\mathrm{s}^{-1}\right)$, a deep LUMO energy level $(-4.07 \mathrm{eV})$, and formed highly transparent pristine films. When applied as an ETL material in an inverted device architecture (ITO/ $\mathrm{NiO}_{x} /$ perovskite $/ \mathrm{PC}_{61} \mathrm{BM} /$ PN6/Ag), PN6 afforded a PCE of $17.27 \%$, which in fact was two times higher than that of the device without the interfacial layer $(8.73 \%)$. It was noted that the PSC device performance was somewhat independent to the interfacial layer thickness, and even at a thickness of $30 \mathrm{~nm}$, a PCE of $15.37 \%$ was achieved. The results demonstrated that a simple molecule based on NDI unit can be used as an efficient ETL material which not only improved all the photovoltaic parameters of PSC devices but represented a new category of water/alcohol soluble interface materials.

Considering the success of PN6, a number of different axially-substituted NDIs were soon reported. Heo reported NDIPM and compared its performance with the conventional PCBM in inverted structured perovskite planar solar cells (Fig. 9). ${ }^{\mathbf{1 1 9}}$ From the design perspective, it was understood that the $\mathrm{N}$ substituted NDI-based compounds are cheaply synthesized by a simple imidation reaction from the commercially-available starting materials and have a comparable solubility and electron mobility to the conventional PCBM. NDI-PM exhibited better electron mobilities of $1.7 \mathrm{~cm}^{2} \mathrm{~V}^{-1} \mathrm{~s}^{-1}$ and $0.72 \mathrm{~cm}^{2} \mathrm{~V}^{-1}$ $\mathrm{s}^{-1}$ in thin film transistors based on single crystals and vacuumdeposited films, respectively, than PCBM $\left(\sim 1 \times 10^{-2} \mathrm{~cm}^{2} \mathrm{~V}^{-1}\right.$ $\mathrm{s}^{-1}$ ). The best inverted $\mathrm{p}-\mathrm{i}-\mathrm{n}$ devices (device architecture: glass/ ITO/PEDOT:PSS/MAPbI ${ }_{3}$ and $\mathrm{FAPbI}_{3-x} \mathrm{Br}_{x} / \mathrm{NDI}-\mathrm{PM} / \mathrm{Al}$ ) afforded $18.4 \%$ and $19.6 \%$ efficiencies, respectively, under 1 sun conditions, which were comparable to those of devices with the PCBM as an ETL (18.9\% and $20.0 \%$, respectively). A $40 \mathrm{~nm}$ thick ETL layer showed the best efficiency under their experimental conditions. The comparable conductivity and charge separation/injection of NDI-PM ETL with the PCBM ETL, validates the strategy that non-fullerene-based NDIs are very promising ETL materials in inverted perovskite devices.

To explore the concept further, Jung reported the use of NDIID (Fig. 9), comprising of an $N$-substituted indane group, synthesised in a one-step procedure from commercially available starting materials. ${ }^{120}$ Though NDI-ID was thermally stable, its solubility in common processing solvents, e.g. methylene chloride, dichloroethane or 1,2-dichlorobenzene was limited, and even lower than the solubility of PCBM at identical concentrations. However, the report claimed that the solubility was enough to fabricate an electron extraction layer using 1,2dichlorobenzene as the processing solvent. The fabricated inverted $\mathrm{p}-\mathrm{i}-\mathrm{n}$ device (device design: glass/ITO/PEDOT:PSS/ $\mathrm{FAPbI}_{3-x} \mathrm{Br}_{x} / \mathrm{NDI}-\mathrm{ID} / \mathrm{Al}$ ) yielded a very high PCE of $20.2 \%$ with an optimized thickness of the ETL being $40 \mathrm{~nm}$. The NDI-IDbased PSCs exhibited very high long-term temporal stability, retaining $90 \%$ of the initial PCE after 500 hours at $100{ }^{\circ} \mathrm{C}$ with 1 sun illumination, without encapsulation. The results suggested that NDI-ID is a promising ETL material which can produce high-performing and thermally stable PSC devices. Continuing their own work, the same research group reported another nonfullerene small-molecule ETL material, NDI-PhE, consisting of axial ( $R$ )-1-phenylethyl (PhE) groups which act as solubilizing groups by reducing molecular symmetry and increasing free volume (Fig. 9). ${ }^{121}$ As a result, the NDI-PhE was found to be more soluble in common processing solvents, such as chlorobenzene and 1,2-dichlorobenzene, and exhibited a good film-forming ability with two-fold solubility compared to fullerene-based ETL materials. The inverted PSCs (glass/ITO/PEDOT:PSS/ $\mathrm{FAPbI}_{3-x} \mathrm{Br}_{x} / \mathrm{NDI}-\mathrm{PhE} / \mathrm{Al}$ ) afforded a comparable PCE of $20.5 \%$, a result that was slightly better than the performance of PCBMbased devices $(20.2 \%)$ of a similar architecture at an optimum thickness of $30 \mathrm{~nm}$. Clearly, the introduction of homochiral groups at the imide nitrogen atoms leading to high solubility in a variety of orthogonal solvents appears to be a good design strategy for simultaneous achievement of high performance and solution-processability in PSC devices. This is further supported by Nakamura's work using the parent naphthalene1,8:4,5-tetracarboxylic diimide (NDI-H) as a transparent ETL material. ${ }^{122}$ Two design factors need to be emphasised. To generate NDI-H, the group first prepared a solution-processable precursor comprising two tert-butoxycarbonyl solubilizing substituents at the imide nitrogens, NDI-Boc, which was then converted to the hydrogen-substituted NDI-H by heating the spin-coated precursor films. The molecular orientation of the hydrogen-bonded NDI-H was examined using two-dimensional grazing incidence X-ray diffraction and p-polarized multipleangle incidence resolution spectrometry. It was observed that the molecular orientation changes from tilted edge-on to the face-on orientation, primarily driven by the formation of intermolecular hydrogen bonds between imide carbonyls and imide hydrogens of neighbouring molecules. As expected, NDI-H was 
insoluble in most of the commonly used solvents. Performance as an ETL material was evaluated by thermal conversion and vacuum deposition on regular-type PSCs fabricated with an ITO/ $\mathrm{PEIE} / \mathrm{NDI}-\mathrm{H} / \mathrm{MAPbI}_{3} /$ spiro-OMeTAD/Au configuration. For comparison, a reference device with $\mathrm{C}_{60}$ as a standard ETL material was also fabricated under the similar conditions. The PSC devices exhibited PCEs of $4.8 \%$ and $10.7 \%$ with thermally converted and vacuum deposited NDI-H layers, respectively. The latter efficiency was comparable to the performance obtained with a $\mathrm{C}_{60}$ ETL in the same device structure (9.3\%). This work suggests that the development of electron-transport materials having shorter absorption wavelengths is an interesting concept and can generate highly efficient ETL materials. A series of such materials was then reported by Li in 2019 using the self-assembly approach to generate electron extraction layers composed of naphthalimide derivatives. ${ }^{\mathbf{1 2 3}}$ Three materials were reported, PN-P, TN-P and NDI-P (Fig. 9), and ETLS constructed via chemical adsorption involving the self-assembly method. All three materials possessed a propionic acid substituent on the nitrogen atom of the imide group to enable their chemical adsorption onto the surface of an indium tin oxide electrode. To modulate the molecular energy levels, different substituents, including electron-rich piperidine (target material: PN-P), 4-trifluoromethylphenyl (target material: TN-P), and electron-deficient imide (target material: NDI-P), were accommodated on the opposite sides. To investigate the potential of these self-assembled electron extraction layers, $n-i-$ p structured devices (configuration: glass/ITO/self-assembledETL/MAPbI ${ }_{3} /$ spiro-OMeTAD/Au) were fabricated. The PCEs were largely improved from $5.4 \%$ (PN-P) to $16 \%$ (NDI-P), suggesting that self-assembly is a low-cost and scalable route to achieve an enhancement of photovoltaic performance. ${ }^{\mathbf{1 2 4}}$

Very recently, a report studying the influence of stereoisomerism on the physical and electronic properties for the diastereoisomers NDI-ID(RR) and NDI-ID(RS), NDI-TN(RR) and NDI-TN(RS), and NDI-PhE(RR) and NDI-PhE(RS) was published (Fig. 9). Interestingly, the authors found that NDI derivatives bearing the RR configuration exhibited more asymmetric crystal characteristics and higher solubility in various organic solvents. The inverted PSCs (ITO/PEDOT:PSS/(FAPbI $)_{x}\left(\mathrm{MAPbBr}_{3}\right)_{1-x} /$ ETL $(\sim 50 \mathrm{~nm}) / \mathrm{Al})$ using both the RR and RS derivatives were fabricated. The RR derivatives afforded better films and high lowest phase transition temperatures, when compared with RS derivatives. The PSCs based on RR derivatives displayed improved performance and higher thermal device stability with less than $10 \%$ degradation of efficiency at $100{ }^{\circ} \mathrm{C}$ for 1000 hours in air, without encapsulation. They concluded that introduction of enantiomeric substitutions results in better solutionprocessability, excellent mobility and surface coverage, higher device performance and enhanced temporal device stability, when compared with mesomeric substitution. ${ }^{125}$

The importance of three-dimensional structure was also highlighted by Ning and coworkers. ${ }^{126}$ Here, they developed NDI-BN, which adopted an "S-shaped, hook-like" structure. NDI_BN was used as a cathode interface layer material to refine the $\mathrm{PC}_{61} \mathrm{BM} / \mathrm{Ag}$ interface in inverted PSCs to improve the stability and performance of devices. NDI-BN showed good planarity along with typical electron transporting property. Inverted PSCs with a configuration of ITO/PTAA/perovskite/ $\mathrm{PC}_{61} \mathrm{BM} / \mathrm{NDI}-\mathrm{BN} / \mathrm{Ag}$ were fabricated and led to PCE of $21.32 \%$ with negligible hysteresis and enhanced stability. The optimal thickness of the NDI-BN layer was found to be only $7 \mathrm{~nm}$, with appropriate LUMO (0.19 eV lower than $\left.\mathrm{PC}_{61} \mathrm{BM}\right)$ and the $\mathrm{HOMO}$ (1.26 $\mathrm{eV}$ lower than $\mathrm{PC}_{61} \mathrm{BM}$ ) levels for efficient electron extraction and beneficial blocking of hole transport. In this work, the team also fabricated reference devices without NDI$\mathrm{BN}$, achieving PCE of $17.71 \%$. The surface morphologies of perovskite/ $\mathrm{PC}_{61} \mathrm{BM}$ and perovskite/ $\mathrm{PC}_{61} \mathrm{BM} / \mathrm{NDI}-\mathrm{BN}$ films were studied using AFM. The AFM analysis revealed a drop in the RMS roughness from $6.74 \mathrm{~nm}$ to $4.56 \mathrm{~nm}$ by incorporating NDI$\mathrm{BN}$, thus, suggesting that a smoother perovskite film is obtained via introducing NDI-BN. Their analysis of using NDI-BN in suppressing interfacial degradation provided evidence that the insertion of NDI-BN layer between $\mathrm{PC}_{61} \mathrm{BM}$ and $\mathrm{Ag}$ can effectively block the pathways of ion migration towards the cathode. It was evident from their study that not only are organic interfacial materials critical to improve device stability and performance, but are important from a molecular perspective of which NDI-based materials are dominant.

3.2.2 Core-substituted NDI (cNDI) derivatives. The first report of cNDI materials used in PSC devices was the compounds NDI3HU-DTYM2 and (DTYM-NDI-DTYA)2, with planar and twisted molecular backbones, respectively (Fig. 9). ${ }^{127}$ Both materials were fabricated in n-channel field effect transistors to achieve electron mobilities up to $3.5 \mathrm{~cm}^{2} \mathrm{~V}^{-1} \mathrm{~s}^{-1}$ (NDI3HU-DTYM2) and $0.45 \mathrm{~cm}^{2} \mathrm{~V}^{-1} \mathrm{~s}^{-1}$ (DTYM-NDI-DTYA)2. The mobilities were also calculated using the space-chargelimited current method, and the electron mobility of (DTYMNDI-DTYA)2 was found to be higher $\left(6.26 \times 10^{-3} \mathrm{~cm}^{2} \mathrm{~V}^{-1} \mathrm{~s}^{-1}\right)$ when compared to NDI3HU-DTYM2 $\left(7.50 \times 10^{-4} \mathrm{~cm}^{2} \mathrm{~V}^{-1} \mathrm{~s}^{-1}\right)$. To study these materials as electron extraction materials, $\mathrm{MAPbI}_{3}$-based PSC devices with inverted $\mathrm{p}-\mathrm{i}-\mathrm{n}$ planar configuration were fabricated. The authors observed that the roughness of the perovskite surface was reduced from about $24.5 \mathrm{~nm}$ to $10.5 \mathrm{~nm}$ and $2.3 \mathrm{~nm}$ with the use of NDI3HU-DTYM2 and (DTYM-NDI-DTYA)2, respectively. The PSC devices using (DTYM-NDI-DTYA)2 as an ETL material produced a PCE of $12.9 \%$, which not only was significantly higher than the devices based on NDI3HU-DTYM2 (8.7\%), but was comparable to the performance of PCBM-based PSC devices (14.3\%). The relatively lower device performance of NDI3HU-DTYM2 was attributed to a lower space-charge limited current (SCLC) electron mobility, lower PL quenching efficiency and rougher interfacial surface. Said later reported two novel, cNDIs, PDPT and PMDPT, as ETL materials (Fig. 9). ${ }^{128}$ The fabricated inverted $\mathrm{p}-\mathrm{i}-\mathrm{n}$ devices (device design: ITO/PEDOT:PSS/ $\mathrm{CH}_{3} \mathrm{NH}_{3} \mathrm{PbI}_{3-x} \mathrm{Cl}_{x} / \mathrm{ETL} / \mathrm{Ag}$ ) with PMDPT afforded a PCE of 9.2\%, whereas the efficiency of PSCs based on PDPT reached $7.6 \%$. This improvement in the efficiency of PMDPT-based devices was attributed to the increased number of nitrogen atoms, which can passivate the electron trap centres on the surface of the perovskite layer. This passivation results in less charge recombination, thus, providing improved device parameters. The thickness of the ETL with different concentrations was also studied and $30 \mathrm{mg} \mathrm{mL}^{-1}$ was 
found to be optimum, yielding superior device outcome and better morphology in terms of covering the perovskite surface without any undesired aggregation. This same research group reported further on two other derivatives, DS1 and DS2 (Fig. 9), as ETL materials in inverted p-i-n PSC devices. ${ }^{129}$ The design of DS1 and DS2 was based on the D-A and D-A-D formats, respectively, where electron rich 4,5-bis(octylthio)-1,3-dithiole2-thione and electron deficient NDI were conjoined. It was envisioned that the incorporation of heteroatoms, such as sulphur, in the molecular design of ETL materials can enhance the interaction between interfacial ETL and perovskite layers via sulphur-iodine or sulphur-lead interactions in inverted $\mathrm{p}-\mathrm{i}-\mathrm{n}$ structures (device architecture: ITO/PEDOT:PSS/ $\mathrm{CH}_{3} \mathrm{NH}_{3}$ $\left.\mathrm{PbI}_{3-x} \mathrm{Cl}_{x} / \mathrm{ETL} / \mathrm{Ag}\right)$. DS1 delivered a PCE of $9.6 \%$, whereas DS2 afforded PCE as high as $11.4 \%$. It was speculated that the presence of extra sulphur atoms in DS2 helped to reduce the energy bandgap and bound more efficiently with the perovskite surface, thus, affording higher device outcome. In further design testing, the same research group further reported two cNDI-based ETL materials, NDI-BTH1 and NDI-BTH2 (ref. 130) bearing two new electron acceptor functionalities, 2-(benzo[d]thiazol-2-yl)acetonitrile and bis(benzo[d]thiazol-2-yl)methane, at the terminals (Fig. 9). Both NDI-BTH1 and NDI-BTH2 displayed good electron mobilities, thermal stability and adequate solubility in orthogonal solvents such as 1,2-dichlorobenzene. The inverted PSC devices were fabricated (device configuration: ITO/PEDOT:PSS/FA $\mathrm{MA}_{1-y} \mathrm{PbI}_{3-x} \mathrm{Cl}_{x} / \mathrm{ETLS} / \mathrm{Ag}$ ) and the NDIBTH1-based devices afforded $13.7 \%$, while the NDI-BTH2based devices yielded $15.4 \%$ efficiencies.

The higher performance of the latter was attributed to its extended conjugation and ambipolar characteristics. In terms of optimal ETL thickness, several solutions with different concentrations were prepared and were used to cast layers on the perovskite surface. The best concentration for casting was found to be $8 \mathrm{mg} \mathrm{mL}^{-1}$ where both the materials afforded the highest performance and with the thickness of $16.9 \mathrm{~nm}$ (NDIBTH1) and $9.6 \mathrm{~nm}$ (NDI-BTH2). This work suggested that highly conjugated, cNDI-based ETL materials have a large scope in the research area of PSC technology and that other acceptor functionalities at the terminals will be worth examining. The latter was particularly true as a series of ETL materials reported by Liu where a new terminal acceptor unit, 2-(2-methyl- $4 H^{-}$ chromen-4-ylidene)malononitrile, was conjoined with the central NDI functionality. The group reported three target ETL materials, NDIF1, NDIF2 and DNDIF3 (Fig. 9). ${ }^{131}$ Broadly speaking, NDIF1, NDIF2 and DNDIF3 were based on the A-A, $\mathrm{A}-\mathrm{A}-\mathrm{A}$ and $\mathrm{D}-\mathrm{A}-\mathrm{A}$ formats, respectively, where the central acceptor was NDI, the terminal acceptor was 2-(2-methyl- $4 H_{-}$chromen-4-ylidene)-malononitrile and the donor was 4,5bis(octylthio)-1,3-dithiole-2-thione. Both absorption and AFM analyses suggested that NDIF2 has strongest molecule-molecule interactions and could aggregate significantly. All three materials were tested as ETLs in inverted planar PSCs with identical film architectures (ITO/PEDOT:PSS/perovskite/ETLs/ $\mathrm{Ag})$. Under the similar testing conditions, NDIF1, NDIF2 and DNDIF3 afforded $15.6 \%, 11.1 \%$, and $11.7 \%$ efficiencies, respectively. Given the strong aggregation in the thin film of
NDIF2, the efficiency drop of $\sim 30 \%$ was justified on that basis, as aggregation of ETLs could affect the performance of PSCs by affecting the roughness of ETL surface, thus, leading to insufficient contact with the metal cathode. This work suggested that it is vital to pay attention to the relationship between design module, molecular interactions and surface morphology when a new, core-substituted ETL material based on NDI format has to be designed.

\section{Discussion on molecular design and processability of ETL materials}

The examples discussed thus far evidently suggest that in terms of molecular design, both polymers and small molecules bearing NDIs can be designed and developed as efficient ETL materials. To date, the number of publications relevant to newly developed small molecules based on NDI are almost identical to the number of publications reporting NDI-based polymers (see Fig. 10).

In terms of device performance, the PCE of devices using small molecule ETLs is slightly better than the efficiency numbers based on polymeric targets, but this is early days and further increases in efficiency need to be seen to break the current ceiling (Fig. 11).

The challenges still lie in defining which principle dominates in which situation. For example, the maximum efficiency for a small molecule target (20.5\%) employed an axially substituted yet simple NDI derivative, NDI-PhE, as an ETL material. ${ }^{121}$ In contrast, the maximum efficiency gained for a polymeric NDI target (18.25\%) was achieved in the conjugated polymer PFNDI, which is orthogonally substituted to NDI-PhE through a cNDI motif, bearing highly soluble branched and straight chain alkyl groups with phosphite esters. ${ }^{114}$ As opposed to the representative polymer examples, the design of small molecules can be easily sub-categorized into axial and core substituted geometries. Although the number of structures based on axial substitution are slightly higher than those of core substituted ones (Fig. 10) and with respect to the highly efficient PSCs using these ETL materials in this review, no clear direction is yet to be made for a preferred design. We further realise that

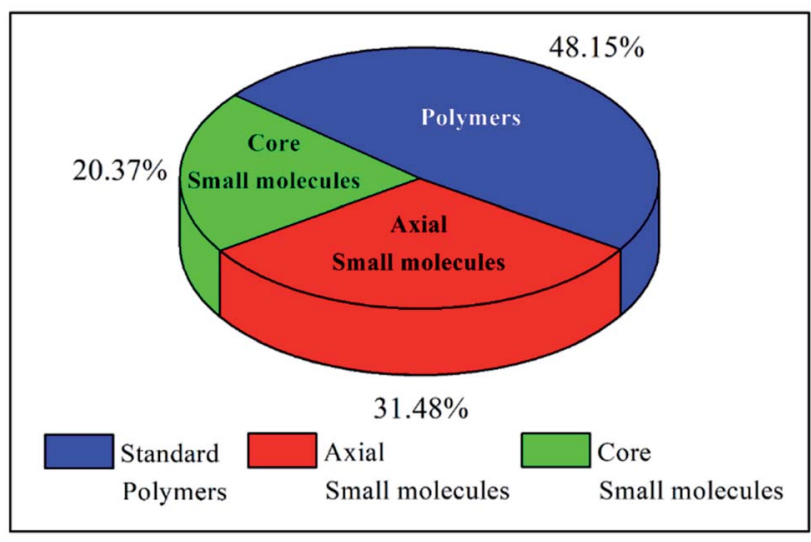

Fig. 10 The ratio of types of reported materials. 
a)

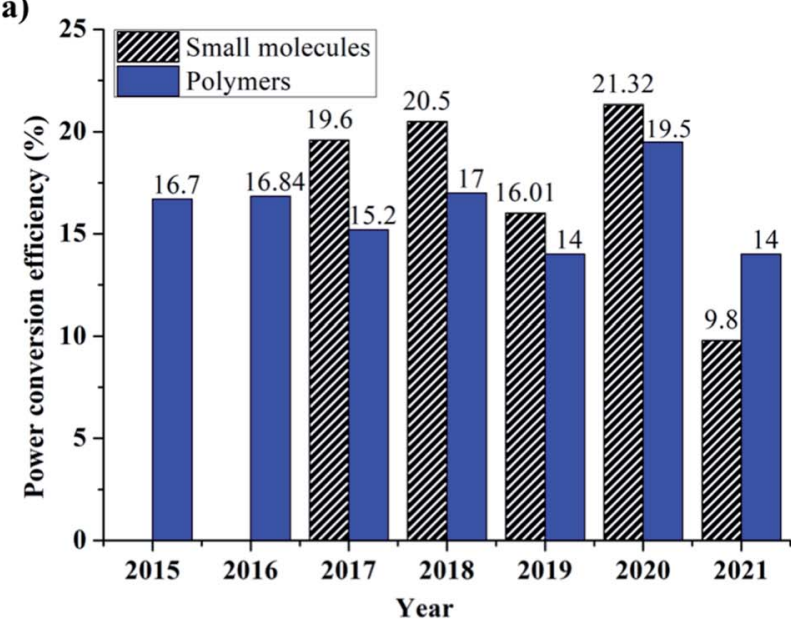

b)

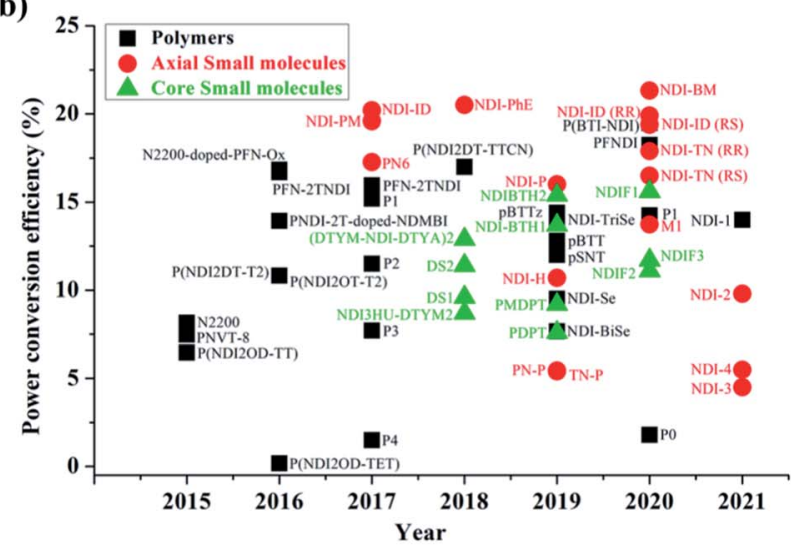

c)

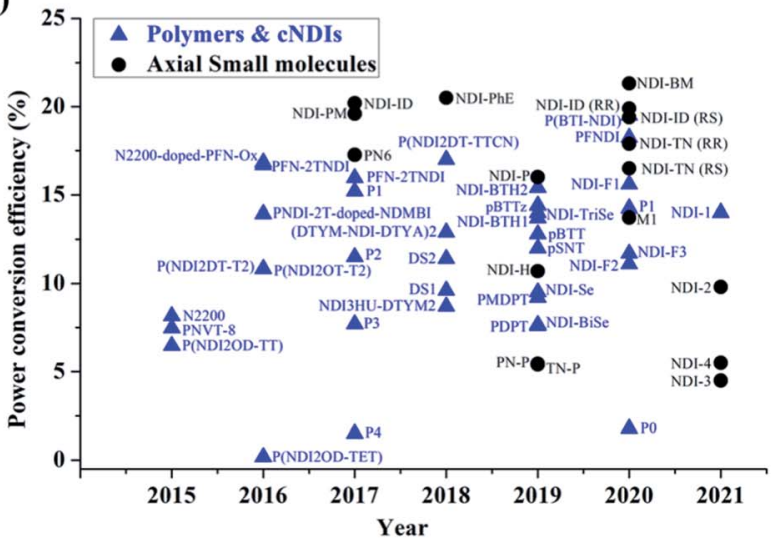

Fig. 11 (a) The maximum PCE numbers reported using polymeric and small molecule targets; (b) an inter-relational plot to represent the comparative PCE values of polymers, and small molecules over time; (c) an inter-relational plot of core vs. axial substitution over the same time scale.

aryl/heteroaryl $N$-substituted NDI derivatives have not been reported for PSCs and this may be due to insolubility that such compounds can be expected to have. For example, the direct connection of functionalities, such as pyridine, pyrimidine and triazine, to name a few, at axial positions has not been reported, though the target materials seem to be promising candidates as ETL materials based on the results reported in this review.
Fig. 11c makes an interesting comparison of axial-substituted small molecules to core substituted examples of both small molecule and polymer types. A less clear differentiation for the need of polymers is seen through this comparison. To date, most organic ETL materials derived from NDIs were fabricated using an inverted device architecture, where the ETL is cast over the perovskite layer. This is reasonable, given the perovskite layer is fabricated using dipolar aprotic solvents, such as dimethylformamide and dimethyl sulfoxide, and the use of orthogonal solvents to lay ETLs on top of the active layer does not affect the surface morphology. A variety of orthogonal solvents, e.g. chlorobenzene, 1,2-dichlorobenzene, toluene, and xylene, have been reported, suggesting that the newly designed materials should possess adequate solubility in a variety of routinely used solvents to promote current protocols. This is one of the major advantages associated with the use of organic ETL materials as they can be structurally tuned to enhance solubility when compared with the conventional $\left(\mathrm{PC}_{61} \mathrm{BM}\right)$ and inorganic (metal oxides) ETL materials. This is particularly true for NDI-based ETL materials as these materials can be designed with a variety of alkyl, alkoxy and polyethylene glycol chains at imide nitrogen atoms to boost their solution processability and hence to exhibit a good film-forming ability as shown throughout (Fig. 8 and 9).

Given the fact that solubility is a vital prerequisite for an ETL material, practically all the ETLs discussed thus far have been deposited via solution-processable techniques, and spincoating in particular. Another method that is worth exploring, albeit less investigated, is vacuum deposition, which can be realised through thermal evaporation. ${ }^{132}$ The advantages may include, (1) excellent surface constancy that may fully cover the perovskite layer, which is useful for textured substrates for example, (2) low ETL surface roughness, (3) enhanced electron mobility, and (4) an ability to process a large number of materials which are otherwise insoluble in orthogonal solvents, thus, avoiding all the environmental unfriendly, toxic solvents. ${ }^{133}$ Nakamura reported the potential of NDI-H as a transparent ETL material ${ }^{122}$ and generated thermally converted and vacuumdeposited electron extraction layers. The PSCs exhibited 4.8\% and $10.7 \%$ efficiencies with thermally converted and vacuum deposited NDI-H layers, respectively. The latter efficiency number was comparable to the efficiency achieved using a $\mathrm{C}_{60}$ ETL in the same device architecture (9.3\%). Moreover, the vacuum-deposited film of NDI-H was observed to have lesser crystallinity than the thermally converted film, suggesting that the low ETL surface roughness can be the key to achieve higher device outcome and that the vacuum deposition technique is worth exploring. The challenge is to ensure the stability and sublimation characteristics of NDIs. According to the literature reviewed, most of the reported materials exhibit the former, i.e. very high decomposition temperature, which, according to some claims, is beneficial for the PSCs, particularly in terms of the devices' temporal stability. Many of the papers reported on device thermal stability and concluded that ETL materials based on NDI format are excellent candidates that can help to improve thermal and overall stability of PSC devices. There is another observation that is be made with respect to the 


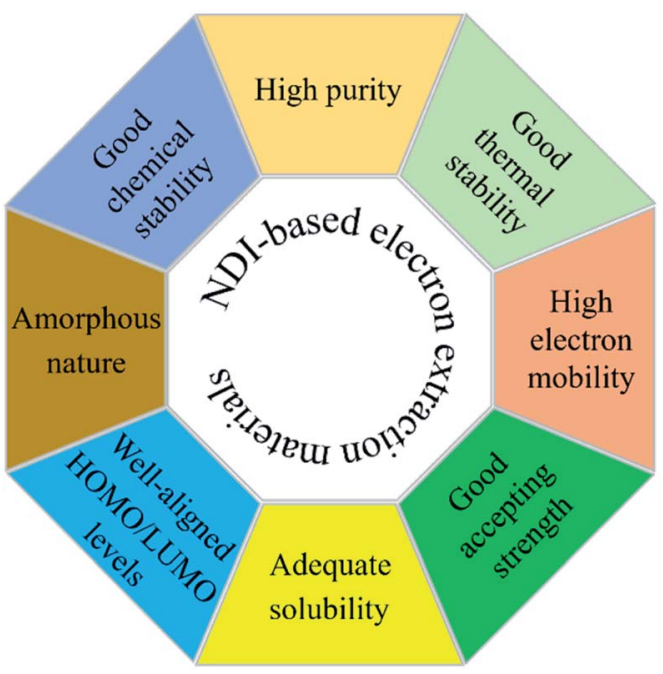

Fig. 12 Features required of efficient, NDI-based n-type materials for use as ETLs.

requirement for high electron mobility in ETL materials. Many of the reported materials also exhibit high electron mobilities in the magnitude range of $10^{-4} \mathrm{~cm}^{2} \mathrm{~V}^{-1} \mathrm{~s}^{-1}$, which is comparable to that of the conventionally used $\mathrm{PC}_{61} \mathrm{BM}\left(\sim 10^{-3} \mathrm{~cm}^{2} \mathrm{~V}^{-1} \mathrm{~s}^{-1}\right)$ as opposed to inorganic ETLs such as $\mathrm{TiO}_{2}$, and appear to be a strong requirement. ${ }^{134}$

Overall, any newly designed organic materials, irrespective of polymers or small molecule, should fulfil a number of requirements to be ideal electron extraction layer materials for PSCs including, high purity, well-tuned HOMO/LUMO energy levels, amorphous nature, good electron mobility, and an ability to interact chemically with the perovskite layer (see Fig. 12). In terms of molecular design, a number of parameters including, (1) extended conjugation with the use of a variety of building blocks such as donor and acceptor, (2) inclusion of lipophilic chains at imide nitrogen atoms, (3) incorporation of functional units containing heteroatoms such as nitrogen and sulfur, and (4) insertion of chiral centres in the solubilising side chains to limit aggregation, can be considered.

\section{Conclusions and outlook}

This review summarizes recent literature reports on electron extraction layer materials based on the NDI molecular structure that have been used as essential components in PSCs, regardless of cell architecture. In this review, we categorized ETL materials into (3.1) polymers and (3.2) small molecules (Fig. 11). The latter was further classified into (3.2.1) axial- and (3.2.2) core-substituted categories. We reviewed the use of a variety of target structures that were generated using the combination of a variety of building blocks, such as donor and acceptors blocks. These blocks are essential components to generate highly conjugated targets, i.e. both polymers or core-substituted small molecules, and their structures were amended to satisfy a number of features, e.g. energy levels, solubility, and thermal stability, required of target materials. ${ }^{81,82}$ It became evident that axial substitution is potentially a better design feature to examine when compared with substitutions based on the core. Generally, core-substituted NDIs are more difficult to produce, require complex purification strategies, and provide lower efficiencies. We also reported device designs, PSC efficiencies, and comparative performance using the conventional ETL materials. We tabulated device parameters including electron mobility values in Tables 1 and 2 .

It is evident from our review that an important area of investigation is the continuation of research on the design and development of inexpensive and efficient ETL materials, and that NDI-based materials show promise as an alternative to the conventional and widely reported materials, e.g. the inorganic metal oxides, $\mathrm{TiO}_{2}, \mathrm{SnO}_{2}$ and $\mathrm{ZnO}$, as well as the fullerene-based $\mathrm{PC}_{61} \mathrm{BM}$ and $\mathrm{C}_{60}$, which can be expensive and processed with the environmentally unfriendly, toxic solvents. Although effective for achieving high cell PCE, the conventionally used $\mathrm{PC}_{61} \mathrm{BM}$ or $\mathrm{C}_{60}$ not only are expensive but suffer from restricted chemical and energetic tuning via structural modification. Such disadvantages encourage researchers to seek alternative structural types which can be easily synthesized, scaled-up and compete with the conventionally used $\mathrm{PC}_{61} \mathrm{BM}$ or $\mathrm{C}_{60}$ by keeping desirable properties such as high electron mobility, solubility, chemical and thermal stabilities and excellent accepting strength. One of such alternative structural types is the NDIbased materials discussed in this review which can certainly exhibit key properties such as good accepting strength, extended conjugation for excellent electron mobility, tuned energy levels and structural diversity. Moreover, these NDIbased targets can be prepared using facile synthetic processes, in high-yield and with high purity, possess excellent electron mobility, and can be structurally tuned to possess complementary energy levels. Furthermore, NDI-based ETLs can be deposited uniformly and reproducibly over large areas using an industrially scalable methodology, e.g. doctor blading or slot die coating, if the commercial application is intended for PSCs. Finally, the ETL materials based on NDI format can have high thermal and environmental stabilities (i.e., no phase transitions below $250{ }^{\circ} \mathrm{C}$ ), thus, leading to long-term temporal stability in PSCs. The requirements of NDI-based target materials are summarised in Fig. 12. This review presents a timely account of recent reports for NDI-based ETL materials and provides insight into the structural aspects that are crucial to designing nextgeneration, highly efficient electron extraction materials for stable PSC devices.

\section{Conflicts of interest}

There are no conflicts to declare.

\section{Acknowledgements}

The authors acknowledge the funding support from the School of Science, Computing and Engineering Technology, Swinburne University of Technology. M. Jameel acknowledges PhD scholarship under the Swinburne University Postgraduate Research Award (SUPRA) program and PhD top-up scholarship from the 
Energy Technologies program, CSIRO Energy, NSW Australia. The CSIRO Division of Manufacturing, Clayton, Victoria Australia is acknowledged for providing support through a Visiting Scientist position for A. G. Authors T. C. J.-Y. and G. J. W. wish to thank the CSIRO Research Office and the Energy Technologies program for project funding, postgraduate scholarships, and fellowship support.

\section{References}

1 H. D. Pham, T. C.-J. Yang, S. M. Jain, G. J. Wilson and P. Sonar, Adv. Energy Mater., 2020, 10, 1903326.

2 I. E. Agency, World Energy Outlook 2018, p. 661, https:// webstore.iea.org/world-energy-outlook-2018.

3 T. M. L. Wigley and S. C. B. Raper, Science, 2001, 293, 451454.

4 J. C. Orr, V. J. Fabry, O. Aumont, L. Bopp, S. C. Doney, R. A. Feely, A. Gnanadesikan, N. Gruber, A. Ishida, F. Joos, R. M. Key, K. Lindsay, E. Maier-Reimer, R. Matear, P. Monfray, A. Mouchet, R. G. Najjar, G.-K. Plattner, K. B. Rodgers, C. L. Sabine, J. L. Sarmiento, R. Schlitzer, R. D. Slater, I. J. Totterdell, M.-F. Weirig, Y. Yamanaka and A. Yool, Nature, 2005, 437, 681-686.

5 T.-J. Kim, Open J. Earthq. Res., 2018, 7, 195-219.

6 A. E. Becquerel, C. R. Acad. Sci., 1839, 561-567.

7 D. M. Chapin, C. S. Fuller and G. L. Pearson, J. Appl. Phys., 1954, 25, 676-677.

8 M. A. Green, K. Emery, Y. Hishikawa, W. Warta and E. D. Dunlop, Prog. Photovoltaics, 2012, 20, 606-614.

9 M. A. Green, Semicond. Sci. Technol., 1993, 8, 1-12.

10 V. P. Equipment, International Technology Roadmap for Photovoltaic (ITRPV) 2019, Germany, 2020.

11 M. A. Green, Sol. Energy Mater. Sol. Cells, 2015, 143, 190197.

12 C. W. Tang, Appl. Phys. Lett., 1986, 48, 183-185.

13 N. S. Sariciftci, L. Smilowitz, A. J. Heeger and F. Wudl, Science, 1992, 258, 1474-1476.

14 Y. Wang, Nature, 1992, 356, 585-587.

15 J. J. M. Halls, C. A. Walsh, N. C. Greenham, E. A. Marseglia, R. H. Friend, S. C. Moratti and A. B. Holmes, Nature, 1995, 376, 498-500.

16 G. Yu, J. Gao, J. C. Hummelen, F. Wudl and A. J. Heeger, Science, 1995, 270, 1789-1791.

17 B. O'Regan and M. Grätzel, Nature, 1991, 353, 737-740.

18 R. F. Service, Science, 2014, 344, 458.

19 C. Eberspacher, J. Ermer, C. Fredric, C. Jensen, R. Gay, D. Pier, D. Willett and F. Karg, Recent Advances in SingleJunction and Tandem Thin Film Modules Based On CuInSe, Dordrecht, 1991.

20 M. Green, Third Generation Photovoltaics, Springer-Verlag Berlin Heidelberg, 2003.

21 G. Conibeer, Mater. Today, 2007, 10, 42-50.

22 M. Green, E. Dunlop, J. Hohl-Ebinger, M. Yoshita, N. Kopidakis and X. Hao, Prog. Photovoltaics, 2021, 29, 3-15.

23 A. Mishra and P. Bäuerle, Angew. Chem., Int. Ed., 2012, 51, 2020-2067.
24 R. V. Hangarge, A. Gupta, A. M. Raynor, D. D. La, A. Bilic, J. Li, D. S. Dalal, R. A. Evans and S. V. Bhosale, Dyes Pigm., 2017, 137, 126-134.

25 S. Economopoulos, G. Itskos, P. Koutentis and S. Choulis, Overview of Polymer and Copolymer Materials for Organic Photovoltaics, ed. C. Brabec, U. Scherf and V. Dyakonov, Wiley Online Library, 2014, pp. 3-26, DOI: 10.1002/ 9783527656912.ch01.

26 C. J. Brabec, Sol. Energy Mater. Sol. Cells, 2004, 83, 273-292.

27 J. Jean, P. R. Brown, R. L. Jaffe, T. Buonassisi and V. Bulović, Energy Environ. Sci., 2015, 8, 1200-1219.

28 P. K. Nayak, S. Mahesh, H. J. Snaith and D. Cahen, Nat. Rev. Mater., 2019, 4, 269-285.

29 S. S. Mali and C. K. Hong, Nanoscale, 2016, 8, 10528-10540.

30 A. Kojima, K. Teshima, Y. Shirai and T. Miyasaka, J. Am. Chem. Soc., 2009, 131, 6050-6051.

31 J. H. Im, C. R. Lee, J. W. Lee, S. W. Park and N. G. Park, Nanoscale, 2011, 3, 4088-4093.

32 H. S. Kim, C. R. Lee, J. H. Im, K. B. Lee, T. Moehl, A. Marchioro, S. J. Moon, R. Humphry-Baker, J. H. Yum, J. E. Moser, M. Grätzel and N. G. Park, Sci. Rep., 2012, 2, 591.

33 M. M. Lee, J. Teuscher, T. Miyasaka, T. N. Murakami and H. J. Snaith, Science, 2012, 338, 643.

34 NREL, Best Research-Cell Efficiencies, https:/www.nrel.gov/ pv/cell-efficiency.html, accessed September, 2020.

35 Editorial, Nat. Energy, 2019, 4, 1, DOI: 10.1038/s41560-0180323-9.

36 M. Jeong, I. W. Choi, E. M. Go, Y. Cho, M. Kim, B. Lee, S. Jeong, Y. Jo, H. W. Choi, J. Lee, J.-H. Bae, S. K. Kwak, D. S. Kim and C. Yang, Science, 2020, 369, 1615-1620.

37 H.-S. Kim, J.-W. Lee, N. Yantara, P. P. Boix, S. A. Kulkarni, S. Mhaisalkar, M. Grätzel and N.-G. Park, Nano Lett., 2013, 13, 2412-2417.

38 A. Yella, L.-P. Heiniger, P. Gao, M. K. Nazeeruddin and M. Grätzel, Nano Lett., 2014, 14, 2591-2596.

39 L. Lin, T. W. Jones, T. C.-J. Yang, N. W. Duffy, J. Li, L. Zhao, B. Chi, X. Wang and G. J. Wilson, Adv. Funct. Mater., 2021, 31, 2008300.

40 T. Leijtens, G. E. Eperon, S. Pathak, A. Abate, M. M. Lee and H. J. Snaith, Nat. Commun., 2013, 4, 2885.

41 J. Song, E. Zheng, J. Bian, X.-F. Wang, W. Tian, Y. Sanehira and T. Miyasaka, J. Mater. Chem. A, 2015, 3, 10837-10844.

42 S. V. Bhosale, M. Al Kobaisi, R. W. Jadhav, P. P. Morajkar, L. A. Jones and S. George, Chem. Soc. Rev., 2021, 1-145, DOI: 10.1002/aenm.201903326.

43 A. Seitkhan, M. Neophytou, M. Kirkus, E. Abou-Hamad, M. N. Hedhili, E. Yengel, Y. Firdaus, H. Faber, Y. Lin, L. Tsetseris, I. McCulloch and T. D. Anthopoulos, Adv. Funct. Mater., 2019, 29, 1905810.

44 S. Wu, Z. Li, M.-Q. Li, Y. Diao, F. Lin, T. Liu, J. Zhang, P. Tieu, W. Gao, F. Qi, X. Pan, Z. Xu, Z. Zhu and A. K. Y. Jen, Nat. Nanotechnol., 2020, 15, 934-940.

45 A. A. Said, J. Xie and Q. Zhang, Small, 2019, 15, 1900854.

46 K. Mahmood, S. Sarwar and M. T. Mehran, RSC Adv., 2017, 7, 17044-17062.

47 M. Cheng, C. Zuo, Y. Wu, Z. Li, B. Xu, Y. Hua and L. Ding, Sci. Bull., 2020, 65, 1237-1241. 
48 U. Bach, D. Lupo, P. Comte, J. E. Moser, F. Weissörtel, J. Salbeck, H. Spreitzer and M. Grätzel, Nature, 1998, 395, 583-585.

49 Q. Wang, C. Bi and J. Huang, Nano Energy, 2015, 15, 275280.

50 Y. Xia and S. Dai, J. Mater. Sci.: Mater. Electron., 2020, 32, 12746-12757.

51 T. Wang, D. Ding, H. Zheng, X. Wang, J. Wang, H. Liu and W. Shen, Sol. RRL, 2019, 3, 1900045.

52 M. S. White, D. C. Olson, S. E. Shaheen, N. Kopidakis and D. S. Ginley, Appl. Phys. Lett., 2006, 89, 143517.

53 F. Eisner, A. Seitkhan, Y. Han, D. Khim, E. Yengel, A. R. Kirmani, J. Xu, F. P. García de Arquer, E. H. Sargent, A. Amassian, Z. Fei, M. Heeney and T. D. Anthopoulos, Sol. RRL, 2018, 2, 1800076.

54 M. K. Siddiki, S. Venkatesan and Q. Qiao, Phys. Chem. Chem. Phys., 2012, 14, 4682-4686.

55 L. Lin, T. W. Jones, T. C.-J. Yang, N. W. Duffy, J. Li, L. Zhao, B. Chi, X. Wang and G. J. Wilson, Adv. Funct. Mater., 2021, 31, 2008300.

56 N. Wang, J. Yu, Y. Zang, J. Huang and Y. Jiang, Sol. Energy Mater. Sol. Cells, 2010, 94, 263-266.

57 Z. He, H. Wu and Y. Cao, Adv. Mater. Interfaces, 2014, 26, 1006-1024.

58 Y. Cai, L. Chang, L. You, B. Fan, H. Liu and Y. Sun, ACS Appl. Mater. Interfaces, 2018, 10, 24082-24089.

59 C. J. Brabec, A. Cravino, D. Meissner, N. S. Sariciftci, T. Fromherz, M. T. Rispens, L. Sanchez and J. C. Hummelen, Adv. Funct. Mater., 2001, 11, 374-380.

60 S. S. Birajdar, S. Brixi, P. S. Rao, R. S. Bhosale, M. A. Kobaisi, A. Gupta, B. H. Lessard, S. V. Bhosale and S. V. Bhosale, ChemistryOpen, 2021, 10, 414-420.

61 S. M. Wagalgave, S. V. Bhosale, R. S. Bhosale, A. L. Puyad, J.-Y. Chen, J.-L. Li, R. A. Evans, A. Gupta and S. V. Bhosale, Mater. Chem. Front., 2019, 3, 1231-1237.

62 D. Srivani, A. Gupta, S. V. Bhosale, A. L. Puyad, W. Xiang, J. Li, R. A. Evans and S. V. Bhosale, Chem. Commun., 2017, 53, 7080-7083.

63 H. D. Pham, T. C.-J. Yang, S. M. Jain, G. J. Wilson and P. Sonar, Adv. Energy Mater., 2020, 10, 2070057.

64 J. Urieta-Mora, I. García-Benito, A. Molina-Ontoria and N. Martín, Chem. Soc. Rev., 2018, 47, 8541-8571.

65 E. Rezaee, X. Liu, Q. Hu, L. Dong, Q. Chen, J.-H. Pan and Z.-X. Xu, Sol. RRL, 2018, 2, 1800200.

66 Z. Zhu, Y. Bai, X. Liu, C.-C. Chueh, S. Yang and A. K. Y. Jen, Adv. Mater. Interfaces, 2016, 28, 6478-6484.

67 K. Yan, J. Chen, H. Ju, F. Ding, H. Chen and C.-Z. Li, J. Mater. Chem. A, 2018, 6, 15495-15503.

68 Q. Hu, W. Chen, W. Yang, Y. Li, Y. Zhou, B. W. Larson, J. C. Johnson, Y.-H. Lu, W. Zhong, J. Xu, L. Klivansky, C. Wang, M. Salmeron, A. B. Djurišić, F. Liu, Z. He, R. Zhu and T. P. Russell, Joule, 2020, 4, 1575-1593.

69 L. Li, H. Chen, Z. Fang, X. Meng, C. Zuo, M. Lv, Y. Tian, Y. Fang, Z. Xiao, C. Shan, Z. Xiao, Z. Jin, G. Shen, L. Shen and L. Ding, Adv. Mater., 2020, 32, 1907257.

70 C. Chen, C. Wu, X. Ding, Y. Tian, M. Zheng, M. Cheng, H. Xu, Z. Jin and L. Ding, Nano Energy, 2020, 71, 104604.
71 M. Zheng, Y. Miao, A. A. Syed, C. Chen, X. Yang, L. Ding, H. Li and M. Cheng, J. Energy Chem., 2021, 56, 374-382.

72 G. Andric, J. F. Boas, A. M. Bond, G. D. Fallon, K. P. Ghiggino, C. F. Hogan, J. A. Hutchison, M. A.-P. Lee, S. J. Langford, J. R. Pilbrow, G. J. Troup and C. P. Woodward, Aust. J. Chem., 2004, 57, 1011-1019.

73 S. V. Bhosale, M. B. Kalyankar, S. V. Bhosale, S. J. Langford, E. F. Reid and C. F. Hogan, New J. Chem., 2009, 33, 24092413.

74 N. Banerji, S. V. Bhosale, I. Petkova, S. J. Langford and E. Vauthey, Phys. Chem. Chem. Phys., 2011, 13, 1019-1029.

75 H. F. Higginbotham, S. Maniam, S. J. Langford and T. D. M. Bell, Dyes Pigm., 2015, 112, 290-297.

76 D. H. Gharib, P. Lock, S. E. Moulton, F. Malherbe and S. J. Langford, ChemNanoMat, 2019, 5, 1303-1310.

77 S. Ali, M. A. Jameel, A. Gupta, S. J. Langford and M. Shafiei, Synth. Met., 2021, 275, 116739.

78 S. Ali, M. A. Jameel, C. J. Harrison, A. Gupta, M. Shafiei and S. J. Langford, Sens. Actuators, B, 2022, 351, 130972.

79 P. S. Rao, A. Gupta, D. Srivani, S. V. Bhosale, A. Bilic, J. Li, W. Xiang, R. A. Evans and S. V. Bhosale, Chem. Commun., 2018, 54, 5062-5065.

80 D. Srivani, A. Agarwal, S. V. Bhosale, A. L. Puyad, W. Xiang, R. A. Evans, A. Gupta and S. V. Bhosale, Chem. Commun., 2017, 53, 11157-11160.

81 S. Maniam, H. F. Higginbotham, T. D. M. Bell and S. J. Langford, Chem.-Eur. J., 2019, 25, 7044-7057.

82 A. Insuasty, S. Maniam and S. J. Langford, Chem.-Eur. J., 2019, 25, 7058-7073.

83 J. Y. Kim, J.-W. Lee, H. S. Jung, H. Shin and N.-G. Park, Chem. Rev., 2020, 120, 7867-7918.

84 N. Li, X. Niu, Q. Chen and H. Zhou, Chem. Soc. Rev., 2020, 49, 8235-8286.

85 R. Wang, M. Mujahid, Y. Duan, Z.-K. Wang, J. Xue and Y. Yang, Adv. Funct. Mater., 2019, 29, 1808843.

86 S. Y. Park, J.-S. Park, B. J. Kim, H. Lee, A. Walsh, K. Zhu, D. H. Kim and H. S. Jung, Nat. Sustain., 2020, 3, 1044-1051.

87 W.-W. Liu, T.-H. Wu, M.-C. Liu, W.-J. Niu and Y.-L. Chueh, Adv. Mater. Interfaces, 2019, 6, 1801758.

88 G. Schileo and G. Grancini, J. Mater. Chem. C, 2021, 9, 6776.

89 X. Tian, S. D. Stranks and F. You, Sci. Adv., 2020, 6, eabb0055.

90 A. Senocrate, G. Y. Kim, M. Grätzel and J. Maier, ACS Energy Lett., 2019, 4, 2859-2870.

91 S. V. Bhosale, C. H. Jani and S. J. Langford, Chem. Soc. Rev., 2008, 37, 331-342.

92 T. D. M. Bell, S. V. Bhosale, C. M. Forsyth, D. Hayne, K. P. Ghiggino, J. A. Hutchison, C. H. Jani, S. J. Langford, M. A. P. Lee and C. P. Woodward, Chem. Commun., 2010, 46, 4881-4883.

93 A. K. Hundal, A. Agarwal, M. A. Jameel, S. Ali, J.-Y. Chen, N. Kaur, L. Jones, J.-L. Li, S. J. Langford and A. Gupta, Sol. Energy, 2020, 195, 223-229.

94 A. K. Hundal, S. Ali, A. Agarwal, M. A. Jameel, L. A. Jones, J.-L. Li, R. A. Evans, S. J. Langford and A. Gupta, J. Phys. 
Chem. Lett., 2021, 10656-10661, DOI: 10.1021/ acs.jpclett.0c03526.

95 M. J. Sung, M. Huang, S. H. Moon, T. H. Lee, S. Y. Park, J. Y. Kim, S.-K. Kwon, H. Choi and Y.-H. Kim, Sol. Energy, 2017, 150, 90-95.

96 Z. Zhang, J. Yuan, Q. Wei and Y. Zou, Front. Chem., 2018, 6, 414.

97 J. Wang and X. Zhan, Trends Chem., 2019, 1, 869-881.

98 K. Rundel, S. Maniam, K. Deshmukh, E. Gann, S. K. K. Prasad, J. M. Hodgkiss, S. J. Langford and C. R. McNeill, J. Mater. Chem. A, 2017, 5, 12266-12277.

99 X. Guo, F. S. Kim, M. J. Seger, S. A. Jenekhe and M. D. Watson, Chem. Mater., 2012, 24, 1434-1442.

100 J. T. E. Quinn, J. Zhu, X. Li, J. Wang and Y. Li, J. Mater. Chem. C, 2017, 5, 8654-8681.

101 Y. H. Ha, J. G. Oh, S. Park, S.-K. Kwon, T. K. An, J. Jang and Y.-H. Kim, Org. Electron., 2018, 63, 250-256.

102 M. Al Kobaisi, S. V. Bhosale, K. Latham, A. M. Raynor and S. V. Bhosale, Chem. Rev., 2016, 116, 11685-11796.

103 W. Wang, J. Yuan, G. Shi, X. Zhu, S. Shi, Z. Liu, L. Han, H.-Q. Wang and W. Ma, ACS Appl. Mater. Interfaces, 2015, 7, 3994-3999.

104 C. Sun, Z. Wu, H.-L. Yip, H. Zhang, X.-F. Jiang, Q. Xue, Z. $\mathrm{Hu}, \mathrm{Z}$. Hu, Y. Shen, M. Wang, F. Huang and Y. Cao, Adv. Energy Mater., 2016, 6, 1501534.

105 C. M. Wolff, P. Caprioglio, M. Stolterfoht and D. Neher, Adv. Mater. Interfaces, 2019, 31, 1902762.

106 S. Shao, Z. Chen, H. H. Fang, G. H. ten Brink, D. Bartesaghi, S. Adjokatse, L. J. A. Koster, B. J. Kooi, A. Facchetti and M. A. Loi, J. Mater. Chem. A, 2016, 4, 2419-2426.

107 Z. Zhu, C.-C. Chueh, G. Zhang, F. Huang, H. Yan and A. K.-Y. Jen, ChemSusChem, 2016, 9, 2586-2591.

108 Y. Guo, W. Sato, K. Inoue, W. Zhang, G. Yu and E. Nakamura, J. Mater. Chem. A, 2016, 4, 18852-18856.

109 D. Li, C. Sun, H. Li, H. Shi, X. Shai, Q. Sun, J. Han, Y. Shen, H.-L. Yip, F. Huang and M. Wang, Chem, 2017, 8, 45874594.

110 T. Jia, C. Sun, R. Xu, Z. Chen, Q. Yin, Y. Jin, H.-L. Yip, F. Huang and Y. Cao, ACS Appl. Mater. Interfaces, 2017, 9, 36070-36081.

111 H. I. Kim, M.-J. Kim, K. Choi, C. Lim, Y.-H. Kim, S.-K. Kwon and T. Park, Adv. Energy Mater., 2018, 8, 1702872.

112 A. A. Said, J. Xie, Y. Wang, Z. Wang, Y. Zhou, K. Zhao, W.-B. Gao, T. Michinobu and Q. Zhang, Small, 2019, 15, 1803339.

113 W. Yan, Z. Wang, Y. Gong, S. Guo, J. Jiang, J. Chen, C. Tang, R. Xia, W. Huang and H. Xin, Org. Electron., 2019, 67, 208214.

114 C. Deng, L. Wan, S. Li, L. Tao, S.-n. Wang, W. Zhang, J. Fang, Z. Fu and W. Song, Org. Electron., 2020, 87, 105959.

115 Y. Shi, W. Chen, Z. Wu, Y. Wang, W. Sun, K. Yang, Y. Tang, H. Y. Woo, M. Zhou, A. B. Djurišić, Z. He and X. Guo, J. Mater. Chem. A, 2020, 8, 13754-13762.
116 S. Valero, A. Cabrera-Espinoza, S. Collavini, J. Pascual, N. Marinova, I. Kosta and J. L. Delgado, Eur. J. Org. Chem., 2020, 2020, 5329-5339.

117 K. Al Kurdi, D. P. McCarthy, D. P. McMeekin, S. O. Furer, M.-H. Tremblay, S. Barlow, U. Bach and S. R. Marder, Mater. Chem. Front., 2021, 5, 450-457.

118 S. Peng, J. Miao, I. Murtaza, L. Zhao, Z. Hu, M. Liu, T. Yang, Y. Liang, H. Meng and W. Huang, J. Mater. Chem. C, 2017, 5, 5949-5955.

119 J. H. Heo, S.-C. Lee, S.-K. Jung, O. P. Kwon and S. H. Im, J. Mater. Chem. A, 2017, 5, 20615-20622.

120 S.-K. Jung, J. H. Heo, D. W. Lee, S.-C. Lee, S.-H. Lee, W. Yoon, H. Yun, S. H. Im, J. H. Kim and O.-P. Kwon, Adv. Funct. Mater., 2018, 28, 1800346.

121 S.-K. Jung, J. H. Heo, D. W. Lee, S.-H. Lee, S.-C. Lee, W. Yoon, H. Yun, D. Kim, J. H. Kim, S. H. Im and O.-P. Kwon, ChemSusChem, 2019, 12, 224-230.

122 T. Nakamura, N. Shioya, T. Shimoaka, R. Nishikubo, T. Hasegawa, A. Saeki, Y. Murata, R. Murdey and A. Wakamiya, Chem. Mater., 2019, 31, 1729-1737.

123 L. Li, Y. Wu, E. Li, C. Shen, H. Zhang, X. Xu, G. Wu, M. Cai and W.-H. Zhu, Chem. Commun., 2019, 55, 13239-13242.

124 S. Y. Kim, S. J. Cho, S. E. Byeon, X. He and H. J. Yoon, Adv. Energy Mater., 2020, 10, 2002606.

125 S.-K. Jung, J. H. Heo, B. M. Oh, J. B. Lee, S.-H. Park, W. Yoon, Y. Song, H. Yun, J. H. Kim, S. H. Im and O.-P. Kwon, Adv. Funct. Mater., 2020, 30, 1905951.

126 J. Ning, Y. Zhu, Z. Hu, Y. Shi, M. U. Ali, J. He, Y. He, F. Yan, S. Yang, J. Miao and H. Meng, Adv. Funct. Mater., 2020, 30, 2000837.

127 C. Ge, W. Wu, L. Hu, Y. Hu, Y. Zhou, W.-S. Li and X. Gao, Org. Electron., 2018, 61, 113-118.

128 A. A. Said, S. M. Wagalgave, J. Xie, A. L. Puyad, W. Chen, Z. Wang, S. V. Bhosale, S. V. Bhosale and Q. Zhang, J. Solid State Chem., 2019, 270, 51-57.

129 D. B. Shaikh, A. A. Said, R. S. Bhosale, W. Chen, S. V. Bhosale, A. L. Puyad, S. V. Bhosale and Q. Zhang, Asian J. Org. Chem., 2018, 7, 2294-2301.

130 D. B. Shaikh, A. Ali Said, Z. Wang, P. Srinivasa Rao, R. S. Bhosale, A. M. Mak, K. Zhao, Y. Zhou, W. Liu, W. Gao, J. Xie, S. V. Bhosale, S. V. Bhosale and Q. Zhang, ACS Appl. Mater. Interfaces, 2019, 11, 44487-44500.

131 W. Liu, D. B. Shaikh, P. S. Rao, R. S. Bhosale, A. A. Said, A. M. Mak, Z. Wang, M. Zhao, W. Gao, B. Chen, Y. M. Lam, W. Fan, S. V. Bhosale, S. V. Bhosale and Q. Zhang, Chem.-Asian J., 2020, 15, 112-121.

132 Y. Vaynzof, Adv. Energy Mater., 2020, 10, 2003073.

133 R. Vidal, J.-A. Alberola-Borràs, S. N. Habisreutinger, J.-L. Gimeno-Molina, D. T. Moore, T. H. Schloemer, I. Mora-Seró, J. J. Berry and J. M. Luther, Nat. Sustain., 2021, 4, 277-285.

134 T. Bak, M. K. Nowotny, L. R. Sheppard and J. Nowotny, J. Phys. Chem. C, 2008, 112, 12981-12987. 